ournal for ImmunoTherapy of Cancer

\title{
Combination of vasculature targeting, hypofractionated radiotherapy, and immune checkpoint inhibitor elicits potent antitumor immune response and blocks tumor progression
}

\author{
Stefano Pierini (DD , ${ }^{1,2}$ Abhishek Mishra, ${ }^{1}$ Renzo Perales-Linares, ${ }^{1}$ \\ Mireia Uribe-Herranz, ${ }^{1,2}$ Silvia Beghi, ${ }^{1}$ Andrea Giglio, ${ }^{1}$ Sergei Pustylnikov, ${ }^{1}$ \\ Francesca Costabile (D) , ${ }^{1}$ Stavros Rafail, ${ }^{2}$ Augusto Amici, ${ }^{3}$ John G Facciponte, ${ }^{2}$ \\ Costantinos Koumenis, ${ }^{1}$ Andrea Facciabene ${ }^{1,2}$
}

To cite: Pierini S, Mishra A, Perales-Linares $\mathrm{R}$, et al. Combination of vasculature targeting, hypofractionated radiotherapy, and immune checkpoint inhibitor elicits potent antitumor immune response and blocks tumor progression. Journal for ImmunoTherapy of Cancer 2021;9:e001636. doi:10.1136/ jitc-2020-001636

- Additional material is published online only. To view please visit the journal online (http://dx.doi.org/10.1136/jitc2020-001636).

$\mathrm{CK}$ and $\mathrm{AF}$ are shared supervision.

Accepted 15 December 2020

\section{Check for updates}

(C) Author(s) (or their employer(s)) 2021. Re-use permitted under CC BY-NC. No commercial re-use. See rights and permissions. Published by BMJ.

For numbered affiliations see end of article.

\section{Correspondence to} Dr Andrea Facciabene; facciabe@pennmedicine.upenn. edu

\section{ABSTRACT}

Background Tumor endothelial marker 1 (TEM1) is a protein expressed in the tumor-associated endothelium and/or stroma of various types of cancer. We previously demonstrated that immunization with a plasmid-DNA vaccine targeting TEM1 reduced tumor progression in three murine cancer models. Radiation therapy (RT) is an established cancer modality used in more than 50\% of patients with solid tumors. RT can induce tumorassociated vasculature injury, triggering immunogenic cell death and inhibition of the irradiated tumor and distant non-irradiated tumor growth (abscopal effect). Combination treatment of RT with TEM1 immunotherapy may complement and augment established immune checkpoint blockade.

Methods Mice bearing bilateral subcutaneous CT26 colorectal or TC1 lung tumors were treated with a novel heterologous TEM1-based vaccine, in combination with RT, and anti-programmed death-ligand 1 (PD-L1) antibody or combinations of these therapies, tumor growth of irradiated and abscopal tumors was subsequently assessed. Analysis of tumor blood perfusion was evaluated by CD31 staining and Doppler ultrasound imaging. Immunophenotyping of peripheral and tumor-infiltrating immune cells as well as functional analysis was analyzed by flow cytometry, ELISpot assay and adoptive cell transfer (ACT) experiments.

Results We demonstrate that addition of RT to heterologous TEM1 vaccination reduces progression of CT26 and TC1 irradiated and abscopal distant tumors as compared with either single treatment. Mechanistically, RT increased major histocompatibility complex class I molecule (MHCl) expression on endothelial cells and improved immune recognition of the endothelium by anti-TEM1 T cells with subsequent severe vascular damage as measured by reduced microvascular density and tumor blood perfusion. Heterologous TEM1 vaccine and RT combination therapy boosted tumor-associated antigen (TAA) cross-priming (ie, anti-gp70) and augmented programmed cell death protein 1 (PD-1)/PD-L1 signaling within CT26 tumor. Blocking the PD-1/PD-L1 axis in combination with dual therapy further increased the antitumor effect and gp70-specific immune responses. ACT experiments show that anti-gp70 T cells are required for the antitumor effects of the combination therapy. Conclusion Our findings describe novel cooperative mechanisms between heterologous TEM1 vaccination and RT, highlighting the pivotal role that TAA cross-priming plays for an effective antitumor strategy. Furthermore, we provide rationale for using heterologous TEM1 vaccination and RT as an add-on to immune checkpoint blockade as triple combination therapy into early-phase clinical trials.

\section{INTRODUCTION}

Radiation therapy (RT) and chemotherapy have traditionally focused on a cell-intrinsic mode of action such as genetic alteration of tumor cells (ie, DNA double-stranded breaks, DNA cross-linking, mitotic catastrophe, and other chromosome abnormalities) leading to cell cycle arrest and cell death. However, recent antitumor strategies have been increasingly focused on tumor cell-extrinsic factors such as induction of systemic antitumor immunity. Radiation-induced immunogenic cell death (ICD) results in stimulation of dendritic cells (DC), ${ }^{1}$ that efficiently engulf tumors and cross-presents tumor-associated antigens (TAAs) to $\mathrm{T}$ cells, eliciting an antitumor response ${ }^{2}$ capable of recognizing distant non-irradiated tumors, known as the abscopal effect. ${ }^{3-5}$ Another important antitumor cell-extrinsic effect induced by RT is radiation-induced damage of the tumor vasculature. $^{6} 7$ Tumor-associated endothelium is significantly altered in response to a single large dose of radiation $(<10 \mathrm{~Gy})$, which in turn makes the tumor microenvironment (TME) hypoxic, acidic, and nutritionally 
deprived, thereby indirectly inducing tumor cell death. ${ }^{89}$ Because of the potential to damage the tumor-associated endothelium and induce ICD, combination treatment of RT together with immunotherapy and/or anti-angiogenic agents can often result in improved therapeutic efficacy. ${ }^{10}$

Active immunotherapies, such as cancer vaccines, aim to reprogram the patient's immune system to recognize and eliminate its own cancer cells via recognition of TAAs. ${ }^{11}$ Immunological targeting of antigens expressed in the tumor-associated endothelial and/or stromal cells, rather than in the tumor cells themselves, is an alternative vaccination approach and has several advantages compared with targeting tumor-based TAAs and include reduced probability of tumor antigen escape variant generation due to the increased genetic stability of the tumor vasculature compared with tumor cells, and improved accessibility by immune cells. ${ }^{12}$ In our previously published work, ${ }^{13}$ we showed that prophylactic immunization with plasmid-DNA encoding the Tem 1 cDNA fused to the minimal domain of the $\mathrm{C}$ fragment of tetanus toxin (TT), used as an immunoenhancer, ${ }^{14-16}$ resulted in complete tumor rejection. When used therapeutically, this approach reduced tumor progression in the CT26 colorectal and TC1 lung cancer models in a T cell-dependent manner. Immunization with the tumor endothelial marker 1 (TEM1) plasmid-DNA construct reduced tumor microvascular density (MVD), decreased tumor blood perfusion, increased hypoxia, and induced potent epitope spreading. ${ }^{13}$

Despite showing encouraging results, therapeutic vaccination with the TEM1 plasmid-DNA did not however result in complete tumor regression. Several strategies to improve vaccination efficacy have been investigated. ${ }^{11} 1718$ Combinations of heterologous modalities of immunization (ie, vaccinating with different vectors encoding the same immunogen) have shown enhanced immune responses to the target antigen. ${ }^{19}$ The rationale behind this strategy is that by using different vectors as boosters, it is possible to bypass the immune response elicited against the primer and also strengthen the immune response against the target antigen. ${ }^{19-21}$

In the current study, we develop a novel recombinant Adenovirus 5 (Ad5) vaccine expressing TEM1-TT fusion protein (TEM1 Ad5) and demonstrate that heterologous priming with TEM1 plasmid-DNA vaccine followed by TEM1 Ad5 vaccine significantly improved TEM1-specific immune responses and antitumor effects compared with either vector alone. ${ }^{2022}$ In vivo, dual treatment with RT and heterologous TEM1 vaccination disrupted the functional vasculature of the irradiated tumor, increased the systemic adaptive immune response, and significantly inhibited the growth of irradiated and non-irradiated (abscopal) tumor as compared with monotherapy. Characterization of the tumor stroma of treated animals reveals that RT promotes immune recognition of tumor-associated vasculature by anti-TEM1 $\mathrm{T}$ cells. Interestingly, blocking the programmed cell death protein 1 (PD-1)/programmed death-ligand 1 (PD-L1) interaction during dual therapy augmented epitope spreading toward the dominant gp70 viral-antigen while paradoxically reducing the frequency of vaccine-induced responses against self TEM1 antigen.

\section{RESULTS \\ TEM1 heterologous prime/boost vaccination increases antitumor effects}

Despite the promising results achieved with the TEM1 plasmid-DNA, ${ }^{13}$ we hypothesized we could further increase vaccination efficacy. DNA prime followed by boosting with viral vectors has been used to enhance immune responses against malaria, ${ }^{23}$ viruses, ${ }^{24}{ }^{25}$ and cancers. ${ }^{26-29}$ We and other have previously demonstrated that heterologous vaccination with plasmid-DNA followed by adenoviral vectors increase the magnitude of the immune response against the target antigen. ${ }^{19} 2022$ Therefore, we cloned the expression cassette of TEM1 (tem1 cDNA fused to TT) into a replication-deficient, human type 5 recombinant adenovirus (TEM1 Ad5) and tested the impact of vaccination using regimens consisting of: (1) three prime-boost injections with the TEM1 plasmid-DNA alone; or (2) three prime-boost injections with TEM1 Ad5 alone; (3) priming with the TEM1 plasmid-DNA followed by two boosts with TEM1 Ad5. Injections were given at weekly intervals and 1 week after the last immunization, splenocytes harvested from vaccinated mice were stimulated with a TEM1 peptide library composed of four peptide's pools (A, B, C, and D) as previously described. ${ }^{13}$ Heterologous prime/boost TEM1 immunization strongly enhanced the vaccine immune response against TEM1 peptides in BALB/c mice (pool A and pool C) and C57BL/6 mice (pool D) compared with vaccination with single vector, as measured by interferon-gamma (IFN- $\gamma$ ) ELISpot assay (figure 1A,B). Similarly, to what previously observed, ${ }^{13}$ no reactivity against pools $\mathrm{A}, \mathrm{B}$, and $\mathrm{C}$ in C57BL6 or against pools B and D in BALB/c after both heterologous and homologous vaccination was observed (data not shown). Interestingly, heterologous TEM1 vaccination also induced a novel response in BALB/c mice directed against an immunogenic peptide (CYALFPRRRTFL; TEM1 $1_{3445}$ ) (online supplemental figure S1A,B), in addition to previously identified peptides. ${ }^{13}$ We then performed a therapeutic vaccination to assess whether the enhanced immunogenicity would also result in improved antitumor effects. Accordingly, we challenged BALB/c mice with CT26 and began vaccination 5 days later. Mice receiving homologous vaccination (ie, TEM1 plasmid-DNA alone or TEM1 Ad5 alone) exhibited delayed tumor progression compared with the control group. Importantly, further reduction of tumor growth was observed in mice receiving heterologous vaccination compared with either single vector (figure $1 \mathrm{C}$ and online supplemental figure S2). Since the heterologous prime/ boost vaccination strategy resulted in enhanced immune responses and improved antitumor effects, we used this immunization protocol in all subsequent experiments. 

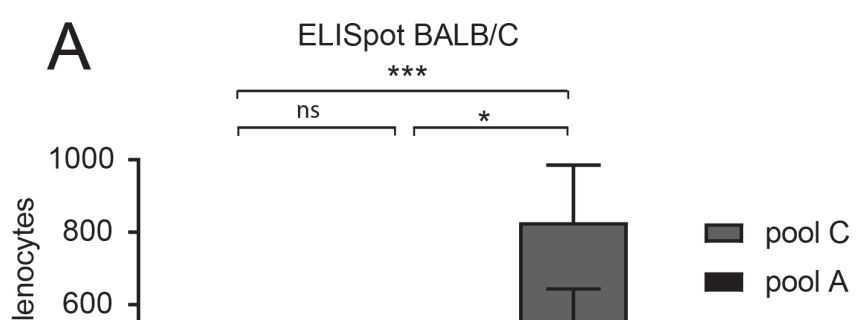
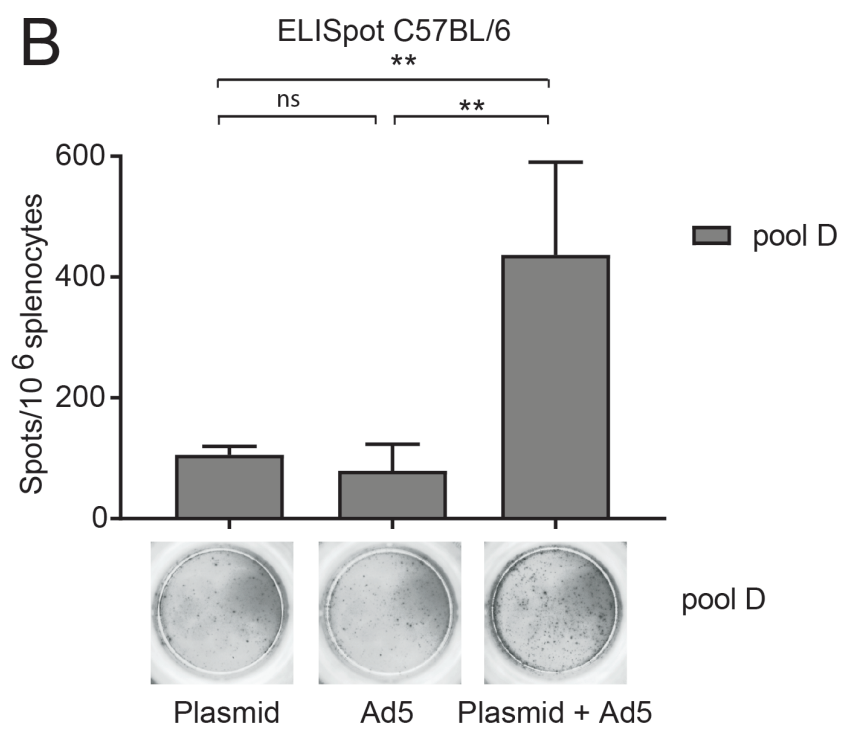

pool A

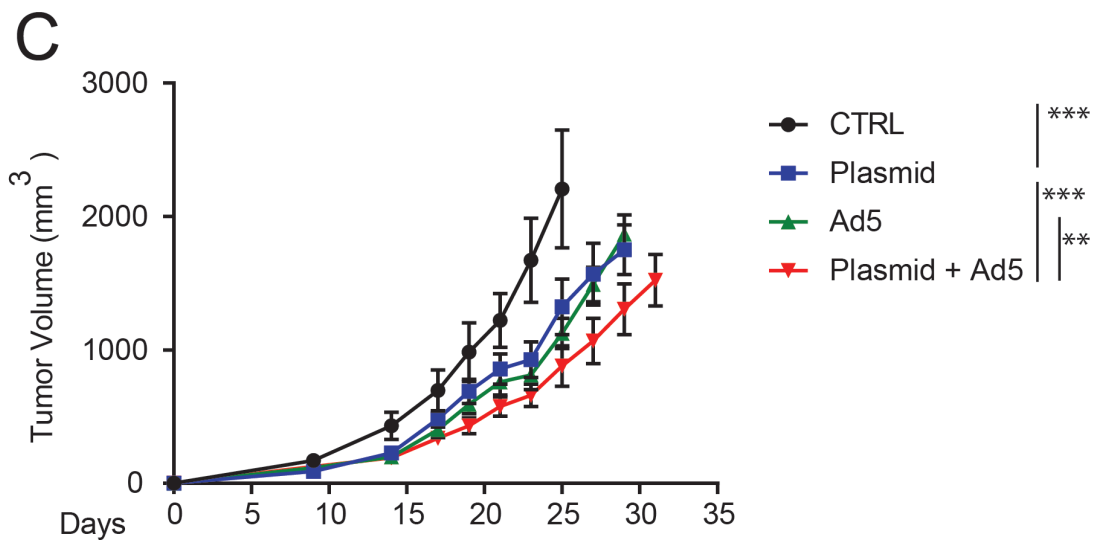

Figure 1 Tumor endothelial marker 1 (TEM1) heterologous prime/boost vaccination increases antitumor effect. BALB/c (A) and C57BL/6 (B) mice were vaccinated with: three prime/boost injections of TEM1 plasmid-DNA (plasmid), three prime/ boost injections of TEM1 Ad5 (Ad5) and prime with TEM1 plasmid-DNA followed by two boosts of TEM1 Ad5 (plasmid+Ad5). Injections were given at weekly intervals and, 1 week after the last immunization, $1 \times 10^{6}$ splenocytes were stimulated overnight with the mouse TEM1 peptide library ${ }^{13}$ and tested by ELISpot. Only reactive pools are shown in the picture. Tukey's multiple comparison tests were performed (BALB/c, Ad5 vs Plas+Ad5 $p=0.025$; Plas vs Plas+Ad5 $p=0.0008$; C57BL/6, Ad5 vs Plas+Ad5 $p=0.008$; Plas+Ad5 vs Plas $p=0.01)$. (C) BALB/c mice were subcutaneously injected with CT26 cells in the lower back and immunization initiated 5 days after tumor inoculation, repeated at weekly intervals for 3 weeks. Tumor growth was monitored throughout the experiment. Differences in tumor volume were evaluated with two-way analysis of variance (ANOVA) test (CTRL vs Plas or Ad5 or Plas+Ad5 $p<0.001$; Plas vs Ad5 $p=n$.s.; Plas+Ad5 vs Ad5 $p=0.0047$; Plas+Ad5 vs Plas $p<0.001$ ). Bar charts illustrate number of interferon-gamma spots. Means \pm SEM are shown from one representative experiment out of three. At least 3 mice/group for the vaccination experiment (A and B) and 7-9 mice/group for the tumor growth experiment (C) were used. ${ }^{*} p<0.05,{ }^{* *} p<0.01,{ }^{* * *} p<0.001$, n.s. non-significant.

\section{Heterologous TEM1 vaccine and early RT results in augmented antitumor effects}

Given the growing interest in combining radiotherapy and immunotherapy for the treatment of solid tumors, ${ }^{103031}$ we investigated whether in vivo irradiation of TC1 and CT26 tumors can enhance the antitumor effects of heterologous TEM1 vaccination. We challenged $\mathrm{BALB} / \mathrm{c}$ and C57BL/ 6 mice with CT26 and TC1 cells, respectively, and then performed RT (21 Gy single dose as previously performed in Uribe-Herranz et $a l^{\beta^{2}}$ ) following either an early $R T$ or late $R T$ schedule (see the Materials and methods section). We performed TEM1 vaccination concurrently with RT, which consisted of priming with the TEM1 plasmid-DNA vaccine followed by RT and two boosts of the TEM1 Ad5 vaccine, repeated at weekly intervals. While TEM1 vaccination as monotherapy significantly reduced CT26 and TC1 tumor progression (figure 2A,C and online supplemental figure S3A,B), RT 

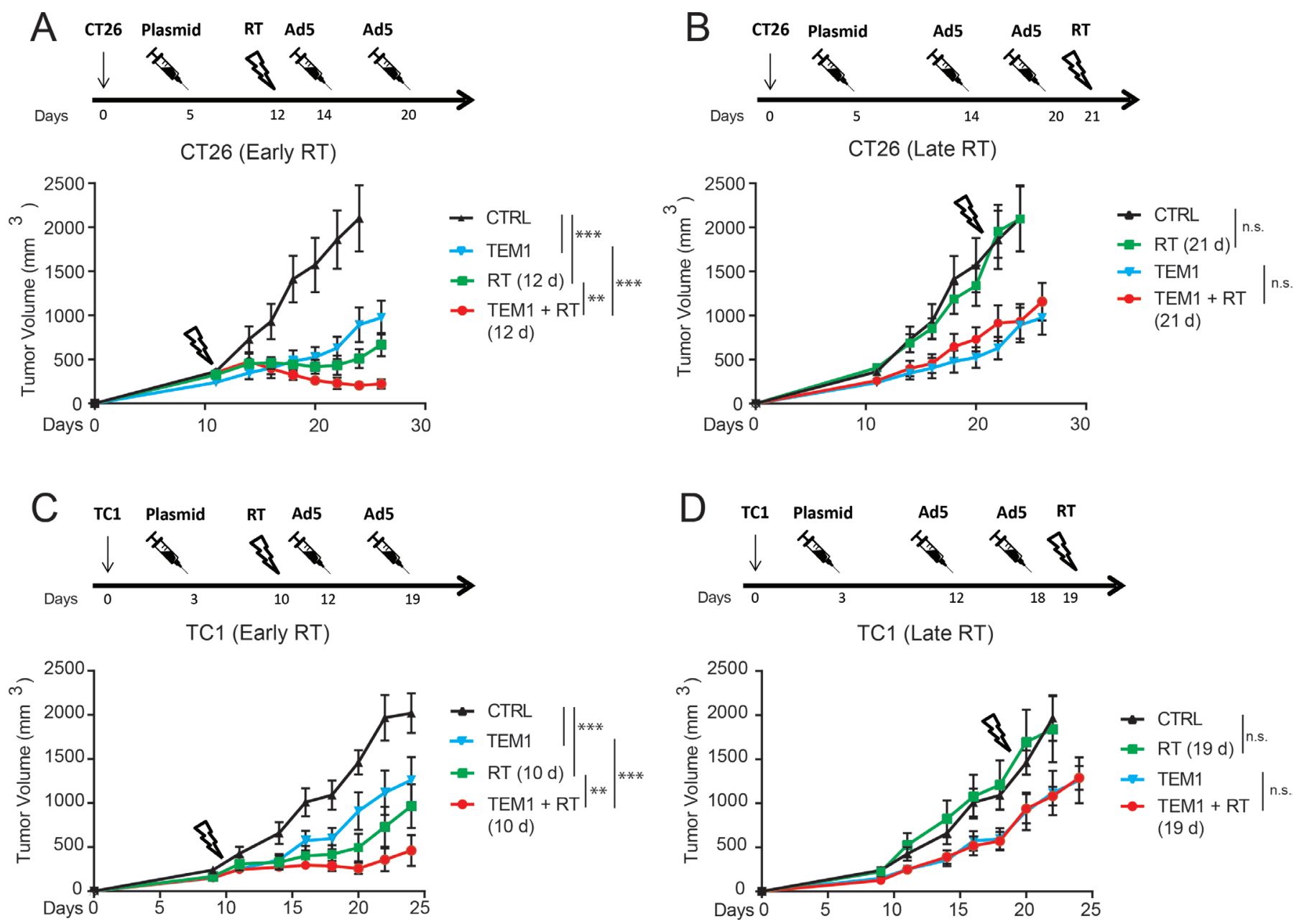

Figure 2 Heterologous TEM1 vaccine and early RT results in augmented antitumor effects. BALB/c (A and B) and C57BL/6 (C and D) mice were subcutaneously injected with CT26 or TC1 cells in the lower back, respectively. Immunization was given 3-5 days after tumor challenge and continued at weekly intervals ( \pm 2 days). Tumor irradiation ( 21 Gy single dose $\left.{ }^{32}\right)$ ) was performed at day 12 in CT26 (200-350 $\mathrm{mm}^{3}$ tumor volume) or at day 10 in TC1 (100-250 $\mathrm{mm}^{3}$ tumor volume) after tumor implantation (early $R T)$ or alternatively on day 21 in CT26 (1600 $\mathrm{mm}^{3}$ mean of tumor volume) or day 19 in TC1 (1450 $\mathrm{mm}^{3}$ mean of tumor volume) (late RT). Tumor growth was monitored throughout the experiment. Differences in tumor volume were evaluated with two-way analysis of variance (ANOVA) test (A, CTRL vs TEM1 or RT or TEM1+RT $p<0.001$; TEM1 vs RT $p=0.21$; TEM1 vs TEM1+RT $p<0.001 ;$ RT vs TEM1+RT $p=0.0032$. B, CTRL vs RT $p=0.62$; TEM1 vs TEM1+RT $p=0.09$. C, CTRL vs TEM1 or RT or TEM1+RT $p<0.001$; TEM1 vs TEM1+RT $p<0.001$; RT vs TEM1+RT $p=0.003$. $D$, CTRL vs RT $p=0.5$; TEM1 vs TEM1+RT $p=0.92$ ). Tumor growth data are representative of one independent experiment out of three with $n>5$ mice/group. Means $\pm S E M$ are shown. ${ }^{\star *} \mathrm{p}<0.01,{ }^{* * *} \mathrm{p}<0.001$, n.s. non-significant. RT, radiation therapy; TEM1, tumor endothelial marker 1.

monotherapy delayed tumor growth specifically when administered early (figure 2A,C and online supplemental figure $\mathrm{S} 3 \mathrm{~A}, \mathrm{~B})$, and not late in the treatment schedule (figure 2B,D and online supplemental figure S3A,B). The heterologous TEM1 vaccine and an early RT schedule resulted in significantly improved antitumor effect in both CT26 and TC1 tumor models, compared with either RT or vaccination alone (figure 2A,C and online supplemental figure S3A,B), whereas the TEM1 vaccine and a late RT schedule did not improve outcomes compared with TEM1 monotherapy (figure 2B,D and online supplemental figure S3A,B). Since administering early RT with heterologous TEM1 vaccination demonstrated attenuated tumor progression, we adhered to the "Plasmid-RTAd5-Ad5" schedule throughout subsequent experiments.

\section{Dual treatment with heterologous TEM1 vaccination and RT reduces abscopal tumor growth}

Since RT can elicit adaptive antitumor immune responses against TAAs, ${ }^{4}{ }^{24}$ we hypothesized that by combining heterologous TEM1 vaccination with RT we could potentiate responses towards abscopal tumors (ie, tumors which are outside the irradiated field). To test this hypothesis, we implemented a two-tumor "abscopal" model in which tumors were injected in both flanks of each mouse at 2-day intervals, and then RT was delivered to only one (primary) tumor. While RT monotherapy did not significantly reduce tumor progression in the CT26 and TC1 models, TEM1 vaccination monotherapy controlled abscopal tumor progression (figure 3B,D and online supplemental figure S4A,B). Notably, the 
A

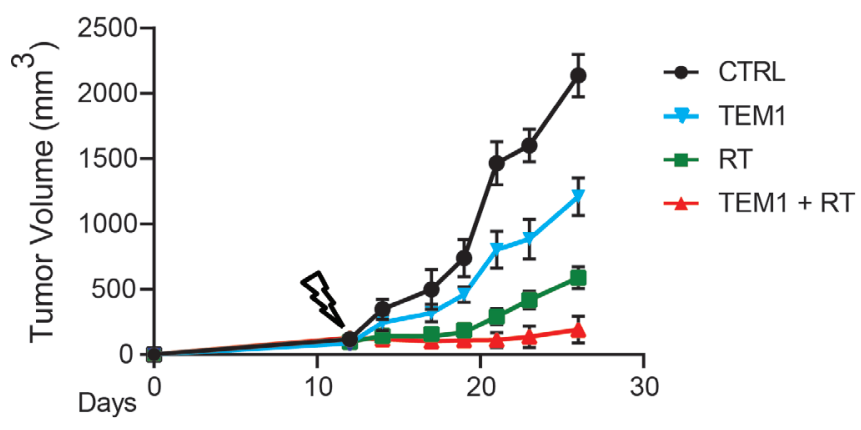

C

TC1 Primary

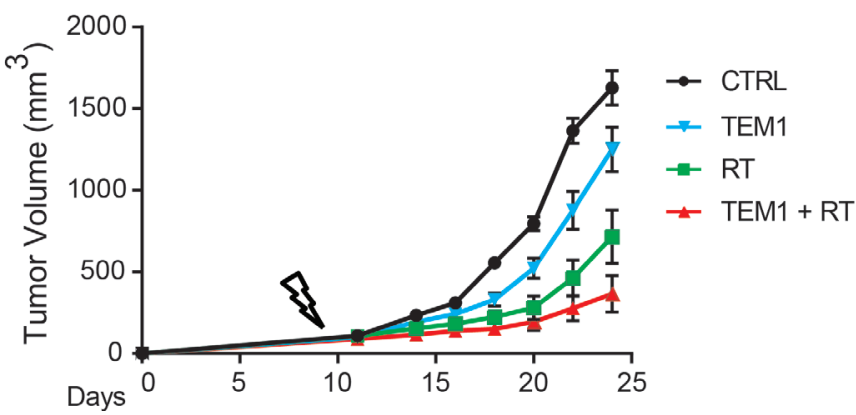

B

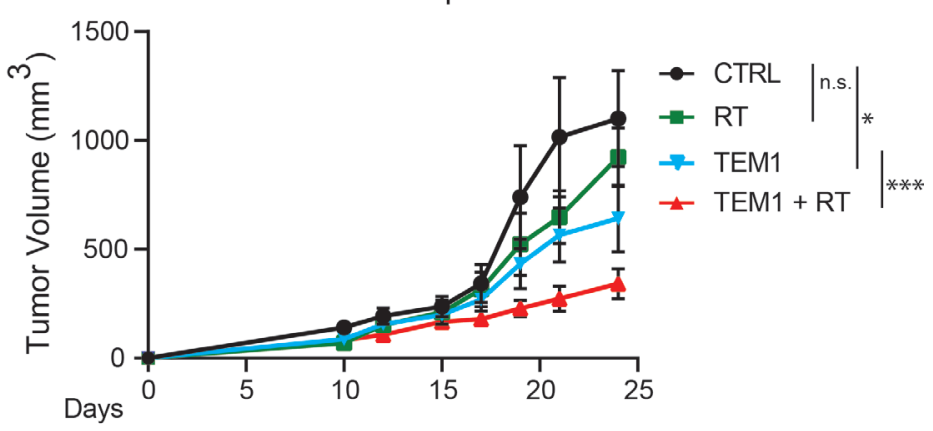

TC1 Abscopal

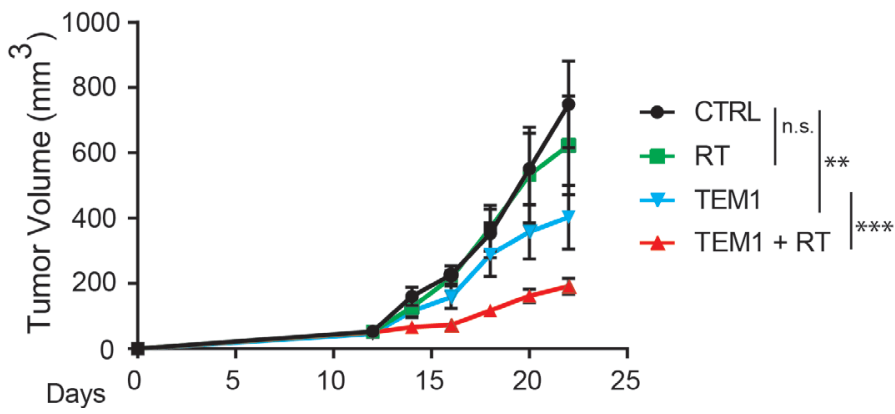

Figure 3 Dual treatment with heterologous TEM1 vaccination and RT reduces abscopal tumor growth. BALB/c (A and B) and C57BL/6 (C and D) mice were injected with CT26 or TC1 cells, respectively. Tumors were injected in both flanks of each mouse at 2-day interval, and then RT was performed only in the irradiated tumor according to the early RT schedule (Plasmid-RT-Ad5$A d 5)$. Growth of both irradiated ( $A$ and $C$ ) and non-irradiated ( $B$ and $D$ ) tumors were followed and data are representative of one independent experiment out of three $(n>5)$. Differences in tumor volume were evaluated with two-way analysis of variance (ANOVA) test $(B)$ and mixed model (D) (B, CTRL vs RT $p=0.14$; CTRL vs TEM1 $p=0.001$; TEM1 vs TEM1+RT $p=0.001$. C, CTRL vs RT $p=0.56$; CTRL vs TEM1 $p=0.004$; TEM1 or RT vs TEM1+RT $p<0.001)$. Means $\pm S E M$ are shown. * $p<0.05$, ** $p<0.01$, ${ }^{* * *} \mathrm{p}<0.001$, n.s. non-significant. $\mathrm{RT}$, radiation therapy; TEM1, tumor endothelial marker 1 .

combination of TEM1 vaccination and RT led to significant reduction of the abscopal (non-irradiated) tumors (figure 3B,D and online supplemental figure S4A,B) in both models compared with monotherapy, suggesting an additive antitumor immune-mediated effect. In summary, timed radiation combined with TEM1-specific heterologous vaccination significantly augments both primary and abscopal antitumor effects.

\section{Dual treatment augments anti-TEM1 T cell recognition of endothelial cells and enhances tumor-associated vascular injury}

To investigate the effects of the dual combination therapy on the tumor vasculature, we first characterized the immune response by screening the reactivity of splenocytes from untreated or TEM1 and TEM1+RT treated mice against TEM1 peptides (pools $\mathrm{A}$ and $\mathrm{C}$ in BALB/C and pool D in C57BL/6). Addition of RT to TEM1 vaccination enhanced T cell recognition of TEM1 epitopes compared with vaccination alone in both CT26 (figure 4A) and TC1 (online supplemental figure S5A) models. Splenocytes from untreated mice or treated with just RT did not show reactivity against TEM1 peptides (online supplemental figure S5B). Due to the observation that cancer cells upregulate major histocompatibility complex class I molecule (MHCI) levels on exposure to radiation, ${ }^{5}$ we asked whether endothelial cells (EC) also respond similarly. Cultured H5V EC, expressing endogenous TEM1, ${ }^{33}$ were irradiated in vitro with 5, 10, or $20 \mathrm{~Gy}$. RT induced a dosedependent increase of Tem 1 mRNA levels (figure 4B) and upregulation of MHCI on the surface of EC (figure 4C). In alignment with the in vitro results, mice receiving RT in vivo (ie, RT alone or TEM1+RT) increased MHCI and Tem1 expression on tumor vasculature (online supplemental figure S5C,D) compared with groups that did not receive RT (ie, TEM1 alone or control). To test whether radiation-induced upregulation of MHCI expression on $\mathrm{H} 5 \mathrm{~V}$ cells results in an increase in immune recognition of the tumor vasculature, we cocultured irradiated $\mathrm{H} 5 \mathrm{~V}$ EC with TEM1-specific T cells and observed enhanced T cell activation in an RT-dose dependent manner, suggesting increased antigen presentation by EC and antigen recognition by TEM1-specific T cells (figure 4D).

Since in the dual combination therapy we observed increased immune reactivity of anti-TEM1 T cells towards their target, we tested if addition of RT to TEM1 vaccination can further damage the tumor vasculature and 

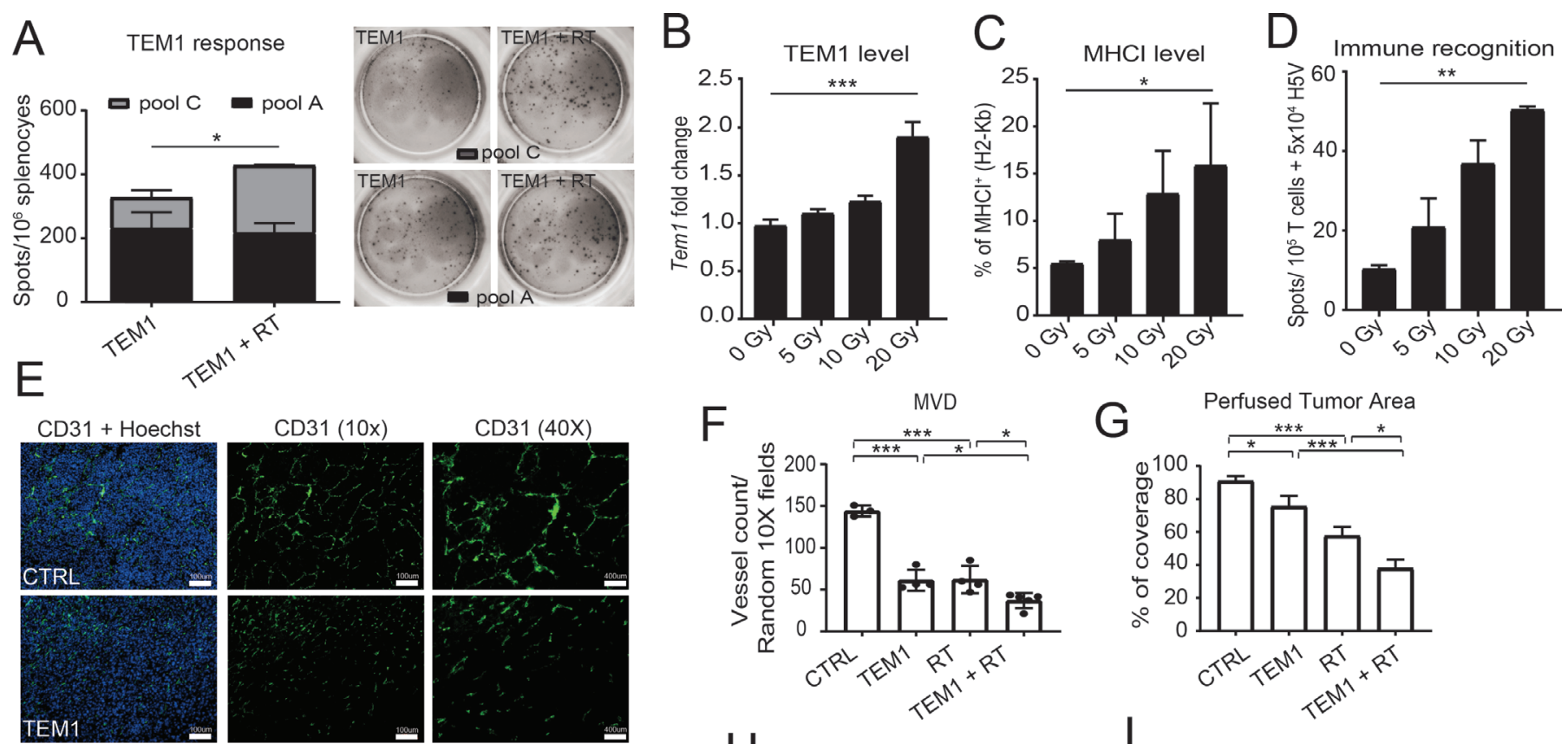
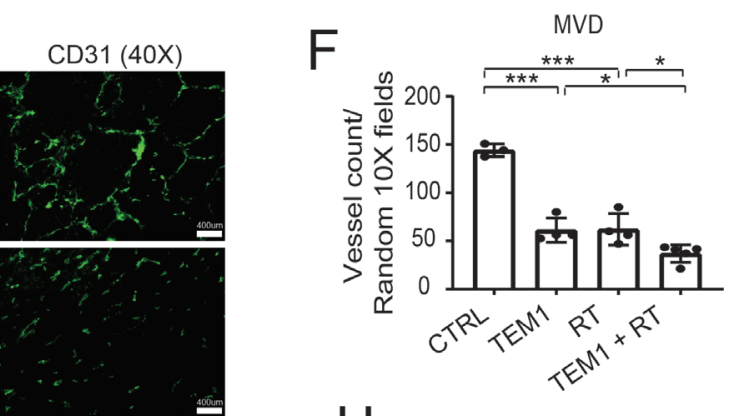

\section{$\mathrm{H}$}

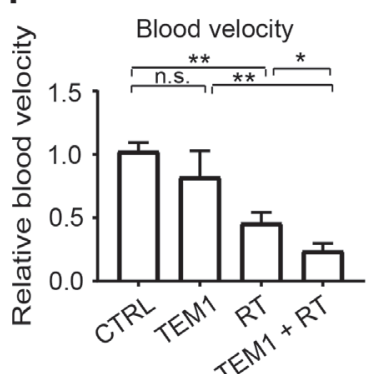

G
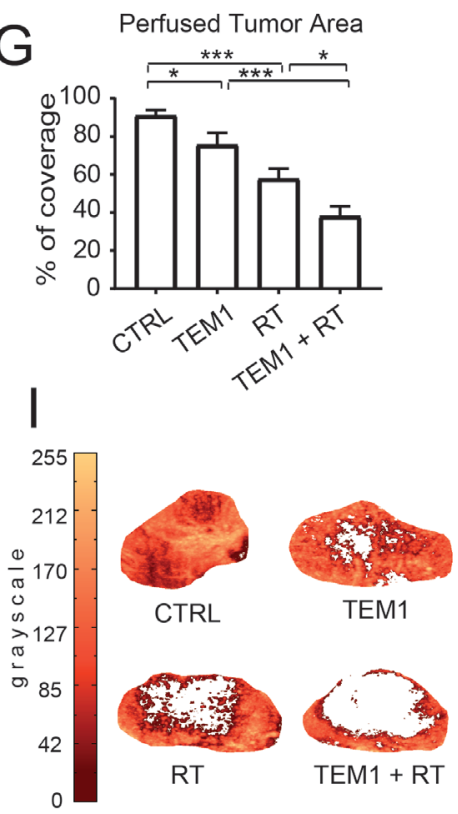

TEM1

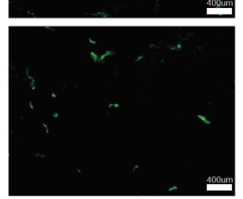

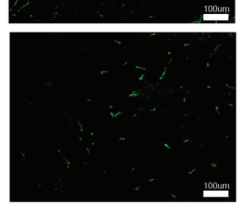

Figure 4 Dual treatment promotes major histocompatibility complex class I molecule (MHCl) expression and immune recognition of endothelial cells by anti-TEM1 T cells enhancing tumor-associated vasculature injury. (A) ELISpot assay performed on splenocytes of treated mice stimulated with TEM1 peptide library shows frequency of peripheral immune response (TEM1 vs TEM1+RT p=0.027). (B-D) In vitro growing H5V cells were exposed to different doses of ionizing radiation and analyzed 24 hours later by real time-PCR (B), by flow cytometry (C). (D) After RT, H5V were cocultured with TEM1-specific T cells isolated from the spleen of vaccinated C57BL6 and ELISpot assay was performed to assess interferon-gamma production. One-way analysis of variance (ANOVA) was performed ( $\mathrm{B}, \mathrm{p}<0.001 ; \mathrm{C}, \mathrm{p}=0.023 ; \mathrm{D}, \mathrm{p}=0.003$ ). (E and F) Quantification of microvascular density (MVD) was achieved by immunofluorescence CD31 staining of CT26 tumors harvest 3 days after RT. Two independent observers quantified MVD by counting CD31 ${ }^{+}$endothelial cells per high-powered field (HPF) (15×22 squares grid was used) at $10 \times$ using ImageJ (CTRL vs TEM1 or RT $p<0.001$; TEM1 vs TEM1+RT $p=0.035$; RT vs TEM1+RT $p=0.028$ ). Mean of 7-10 fields per mouse and six mice group/were used. (E) Representative if slides at 10× and 40× magnification. (G) Bar charts show \% of blood perfused areas of the tumor and relative blood velocity $(\mathrm{H})$ as measured by Doppler image analysis (G, CTRL vs TEM1 $p=0.05$; CTRL vs RT $p<0.001$; TEM1 vs TEM1+RT $p<0.0001$; RT vs TEM1+RT $p=0.041$. H, CTRL vs TEM1 $p=0.62$, CTRL vs RT $p=0.007$, TEM1 vs TEM1+RT $p=0.009$; RT vs TEM1+RT $p=0.043$ ). (I) Representative maximum intensity projections of tumor volumes after treatment with TEM1 alone, RT alone or combination of TEM1+RT measured at day 17 after tumor challenge (2 days after RT). Data were obtained with two-dimensional high-frequency ultrasound. Red color represents the lowest power Doppler intensity while yellow represents the highest power Doppler intensity. Means $\pm S E M$ are shown from 1 experiment with 4 mice/group. Tukey's multiple comparison tests were performed. ${ }^{*} p<0.05,{ }^{* *} p<0.01$, ${ }^{* *} p<0.001$, n.s. nonsignificant. RT, radiation therapy; TEM1, tumor endothelial marker 1.

alter blood perfusion in the hyper-vascularized CT26 model than either modality alone. To ensure that tumors of the same size were analyzed, treated mice were euthanized later than untreated mice so that tumors could be collected at similar volumes $\left(\sim 300 \mathrm{~mm}^{3}\right)$ and then cryopreserved. Quantification of $\mathrm{CD}^{+} 1^{+} \mathrm{EC}$ per high-power field revealed that mice receiving either TEM1 vaccination or RT had lower MVD than the untreated control group. Dual treatment with both TEM1 vaccination and RT further reduced MVD compared with monotherapy (figure $4 \mathrm{E}, \mathrm{F}$ ). We then measured functionality of the tumor vasculature by assessing blood perfusion (percentage of total tumor area) and blood velocity (time to perfuse a given area) using Doppler ultrasound imaging when tumors reached a volume of $\sim 600 \mathrm{~mm}^{3}$. To ensure similar tumor volumes, tumor inoculation was delayed by 3 days in the control groups, and mice receiving RT (ie, RT alone or TEM1+RT) were irradiated 
when the tumors reached a mean volume of $500 \mathrm{~mm}^{3}$ (approximately day 15). Gas-filled microbubbles were injected as a contrast agent to highlight the tumor blood vessel. On day 17 post tumor challenge ( 2 days after RT), contrast-enhanced images showed a reduction in blood perfusion in both TEM1-treated and RT-treated groups compared with the untreated control group. Notably, total blood perfusion and velocity were further reduced in the combination group compared with either group receiving monotherapy (figure 4G-I). Together, these data suggest that TEM1 vaccination and RT independently negatively impact the functionality of the tumor vasculature by reducing tumor MVD and tumor blood perfusion. Addition of RT to TEM1 vaccination augments the TEM1specific $T$ cell response, increases endothelium MHCI expression and immune recognition by TEM1-specific $\mathrm{T}$ cells, compared with vaccination alone, leading to much greater impact on the tumor vasculature.

\section{Dual treatment enhances DC/macrophage activation, promotes TAA cross-priming and boosts T cells infiltration}

Since we observed augmented systemic antitumor effects by combining heterologous TEM1 vaccination with early RT, we hypothesized that combination therapy would result in enhanced epitope spreading, ${ }^{34}$ a phenomenon induced by TEM1 vaccination ${ }^{13}$ as well as RT. ${ }^{1}$ We used the $\mathrm{GP} 70_{423-431}$ (AH1) and E7 ${ }_{49-57}$ peptides, the immunodominant MHCI-associated epitopes for CT26 and TC1, respectively. ${ }^{35}{ }^{36}$ Splenocytes isolated from CT26 and TC1 tumor-bearing mice that underwent dual treatment were stimulated overnight with the $\mathrm{AH} 1$ and $\mathrm{E} 7_{49-57}$ peptides and $\mathrm{T}$ cell activation was assessed by ELISpot. Dual combination treatment of TEM1 vaccination with early RT enhanced immune responses against gp 70 and $\mathrm{E} 7$ peptides compared with either monotherapy, suggesting increased epitope spreading (figure 5A and online supplemental figure S6A). Adding late RT to the vaccination failed to increase E7 peptide response in the TC1 model (online supplemental figure S6B). We next inquired if anti-gp70 specific $\mathrm{T}$ cells would functionally recognize irradiated CT26 cells. Gp70-specific T cells (refer to IgE-gp70-TT vaccine in the Materials and methods section) were cultured with CT26 and T cell activation was assessed by IFN- $\gamma$ ELISpot. Remarkably, RT strongly increased MHCI expression (online supplemental figure S6C) and target cell recognition (figure 5B) in a dose-dependent manner. $\mathrm{T}$ cell activation was abrogated when a $\beta 2$ microglobulin (B2M) knockdown CT26 cell line (CT26 B2M ${ }^{-}$) was used, indicating that $\mathrm{T}$ cell recognition was MHCI-dependent (figure 5B).

$\mathrm{T}$ cells contribute to both RT-induced and vaccineinduced antitumor responses, ${ }^{37} 38$ we therefore quantified T cell infiltration in the CT26 model and observed a significant increase in $\mathrm{CD}^{+} \mathrm{T}$ cell infiltration in the abscopal tumor of mice receiving the combination therapy compared with individual therapies (online supplemental figure S6D). Tetramer analysis (online supplemental figure S7) on digested tumor cell suspensions revealed increased infiltration of gp70-specific and E7-specific $\mathrm{T}$ cells in CT26 and TC1 abscopal tumors (figure 5C and online supplemental figure S6E) and TC1 primary tumor (online supplemental figure S6F) on combination therapy compared with TEM1 or RT alone. To pinpoint the cell types that may contribute to the enhanced adaptive immunity, we characterized single-cell suspensions of draining lymph nodes (LNs) and tumors, and specifically examined the myeloid compartment, which includes DC and macrophage. Flow cytometry analysis (online supplemental figure S7) showed increased expression of MHCII and CD40 activation markers on CD11c ${ }^{+}$ cells-containing both DC and macrophage ${ }^{39}{ }^{40}$-in the draining LNs (figure 5D) and tumors (figure 5E) from mice receiving combination therapy compared with monotherapy. To determine whether the CD11c positive population specifically contribute to $\mathrm{T}$ cell activation, $\mathrm{CD} 11 \mathrm{c}^{+}$cells were sorted from collagenase-digested LN cell suspension, pulsed with AH1 peptide, and cocultured with gp70-specific T cells. As hypothesized, CD11c positive cells originating from mice that received the combination therapy had significantly increased capacity to activate gp70-specific $\mathrm{T}$ cells relative to the $\mathrm{CD} 11$ c positive cells from mice that received monotherapy (figure $5 \mathrm{~F}$ ). Cumulatively, these results suggest a potential mechanism in which radiation activates $\mathrm{CD} 11 \mathrm{c}^{+}$cells that in turn contributes to TAA cross-priming and promotes antigenspecific (ie, gp70 and E7) T cell infiltration within the tumor.

\section{Inhibition of PD-1/PD-L1 axis enhances the antitumor effects of dual therapy}

Previous studies have shown that RT can also stimulate inhibitory pathways, such as the PD-1/PD-L1 axis, and hence contribute to immunosuppression. ${ }^{41}$ Therefore, we assessed surface levels of PD-L1 on cultured CT26 and H5V cell lines in response to 5, 10, or $20 \mathrm{~Gy}$ of ionizing radiation and observed a dose-dependent increase on both cell lines (online supplemental figure S8A,B). In vivo, increased levels of PD-L1 mRNA expression was observed in irradiated tumors (ie, RT alone or TEM1+RT) compared with tumors that did not receive RT (ie, untreated CTRL or TEM1 alone) (figure 6A). We observed by flowcytometry (online supplemental figure S7) higher levels of PD-1 expression on $\mathrm{CD}^{+} \mathrm{T}$ cells within tumors of irradiated mice compared with non-irradiated mice (figure 6B). We also examined $I F N-\gamma$ cDNA levels in tumors (online supplemental figure S8C) and found that combination therapy increased IFN- $\gamma$ expression compared with each monotherapy.

Due to the observed increase in PD-1/PD-L1 expression, we reasoned that blocking the PD-1/PD-L1 axis with immune checkpoint inhibitors (ICIs) therapy could further enhance antitumor responses in our model. Accordingly, the therapeutic protocol of heterologous TEM1 vaccination with early RT was combined with administration of an anti-PD-L1 antibody ( $\alpha$ PDL1) starting at day 12, two times per week, for a total of 


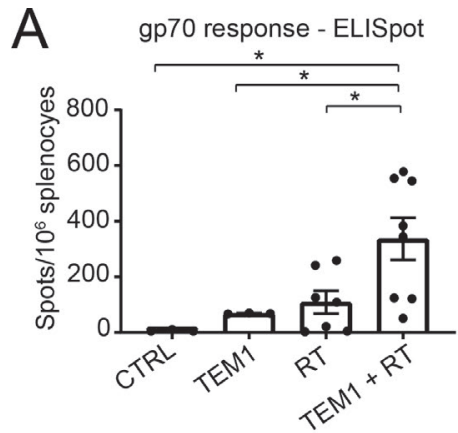

B
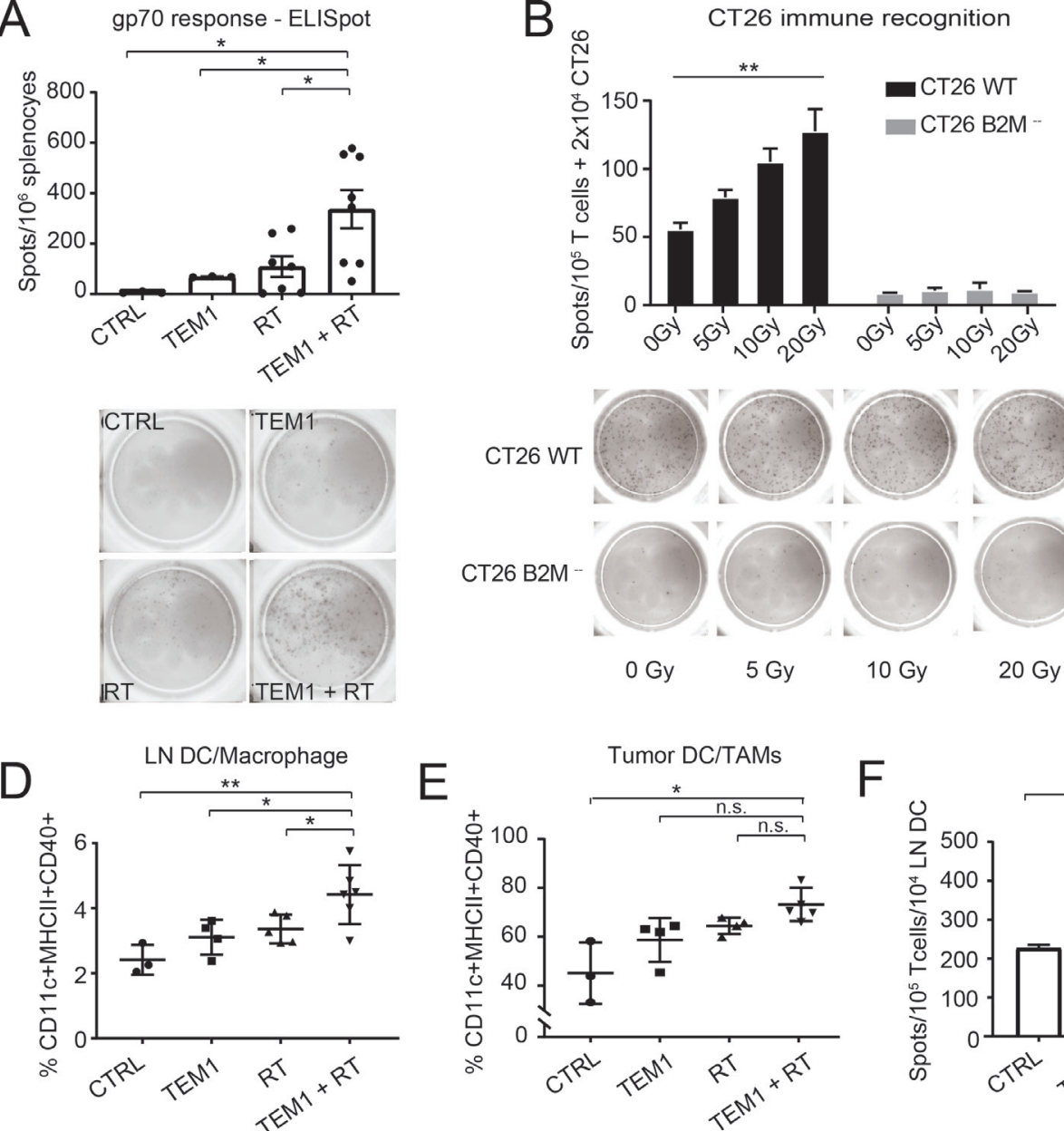

$E$

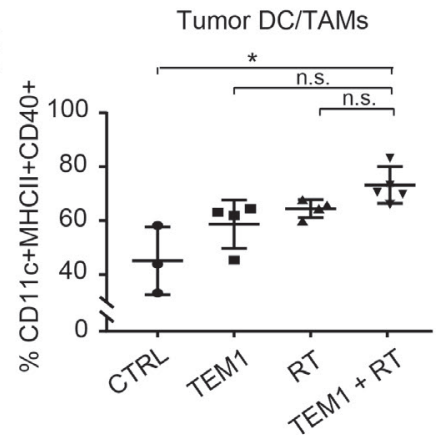

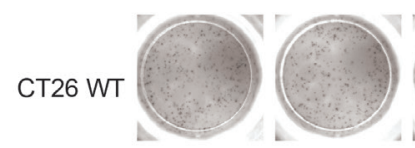

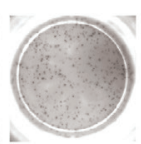

$\mathrm{F}$
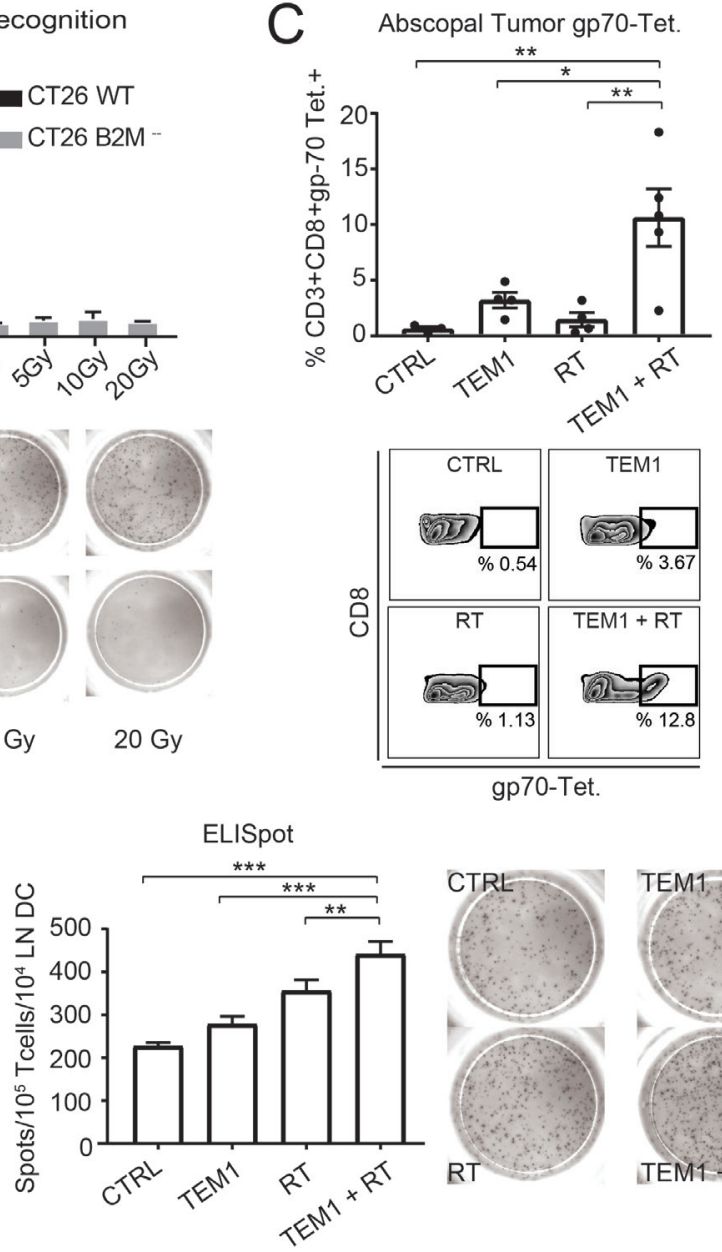

Figure 5 Dual treatment enhances $\mathrm{DC} /$ macrophage activation, promotes tumor-associated antigen cross-priming and boost T cells infiltration. (A and C) CT26-bearing mice were sacrificed to harvest spleen and tumors. (A) Splenocytes from untreated (CTRL) mice or treated with TEM1, RT and dual therapy (TEM1+RT) were stimulated with $A H 1$ peptide and assayed for interferon-gamma (IFN- $\gamma$ ) ELISpot. (C) Quantification by flow cytometry shows percentage of gp70-specific T cells (gp70Tet.) infiltrating the abscopal tumor (CTRL vs TEM1+RT $p=0.009$; TEM1 vs TEM1+RT $p=0.035$; RT vs TEM1+RT $p=0.009$ ). Percentage of total CD8 ${ }^{+} \mathrm{T}$ cells is reported. Representative flow cytometry plots are shown. (B) In vitro growing CT26 cells or CT26 B2M-deficient cells (CT26 B2M) were exposed to different doses of ionizing radiation and cocultured with gp70-specific T cells isolated from the spleen of BALB/c (vaccinated against gp70; refer to IgE-gp70-TT vaccine in material and methods) and ELISpot assay was performed to assess IFN- $\gamma$ production. One-way analysis of variance (ANOVA) tests were performed $(p=0.003)$. ( $D$ and $E)$ Mice receiving therapies were sacrificed 4 days after $R T$ to harvest organs. Flow cytometry analysis performed on digested lymph nodes (D) and primary tumors (E) suspensions shows expression of MHCII+CD40+ markers, indicative of $D C$, macrophage and tumor-associated macrophage (TAM) activation ( $D, C T R L$ vs TEM1+RT $p=0.002$; TEM1 vs TEM1+RT $p=0.02$; RT vs TEM1+RT $p=0.044$. $E$, CTRL vs TEM1+RT $p=0.002$ ). (F) ELISpot assay shows ability of $C D 11 c^{+} D C /$ macrophage sorted from tumor-draining lymph nodes of treated mice to mediate activation of $\mathrm{gp} 70^{+} \mathrm{T}$ cells in the presence of $A H 1$ peptide (CTRL vs TEM1 +RT $p<0.0001$; TEM1 vs TEM1+RT $p<0.0001$; RT vs TEM1+RT $p=0.0063$ ). Means $\pm S E M$ are shown. Each dot represents a mouse. Tukey's multiple comparison tests were performed. ${ }^{*} p<0.05,{ }^{* *} p<0.01,{ }^{* \star *} p<0.001$, n.s. non-significant. DC, dendritic cells; LN, lymph node; RT, radiation therapy; TEM1, tumor endothelial marker 1.

three injections (figure 6C). Although treatment with RT alone did not significantly impede the growth of abscopal tumor, treatment with either TEM1 vaccination alone or with $\alpha$ PD-L1 alone, both systemic in nature, significantly inhibited abscopal tumor progression (figure $6 \mathrm{D}, \mathrm{E}){ }^{42}$ Combination treatment with RT and $\alpha$ PD-L1 impaired the growth of the abscopal tumor to a degree similar to that of the combination treatment with TEM1 vaccination and RT. Surprisingly, combining anti-PD-L1 treatment with the TEM1 vaccine did not improve the antitumor effect compared with just TEM1 vaccination. Importantly, triple combination therapy with TEM1 vaccine, RT, and $\alpha$ PD-L1 further reduced abscopal tumor progression compared with both dual treatments (ie, TEM1+RT or RT $+\alpha P D-L 1$ ) (figure 6D,E). Long-term monitoring for survival in the one-tumor model revealed that dual treatments with TEM1+RT or RT $+\alpha$ PD-L1 significantly extended survival compared with individual therapies (TEM1 alone, RT alone and $\alpha$ PD-L1 alone) (figure 7A). These treatments induced rejection of tumors in $60 \%$ and $42.9 \%$ of the mice, respectively. Triple combination treatment with TEM1 vaccine, 

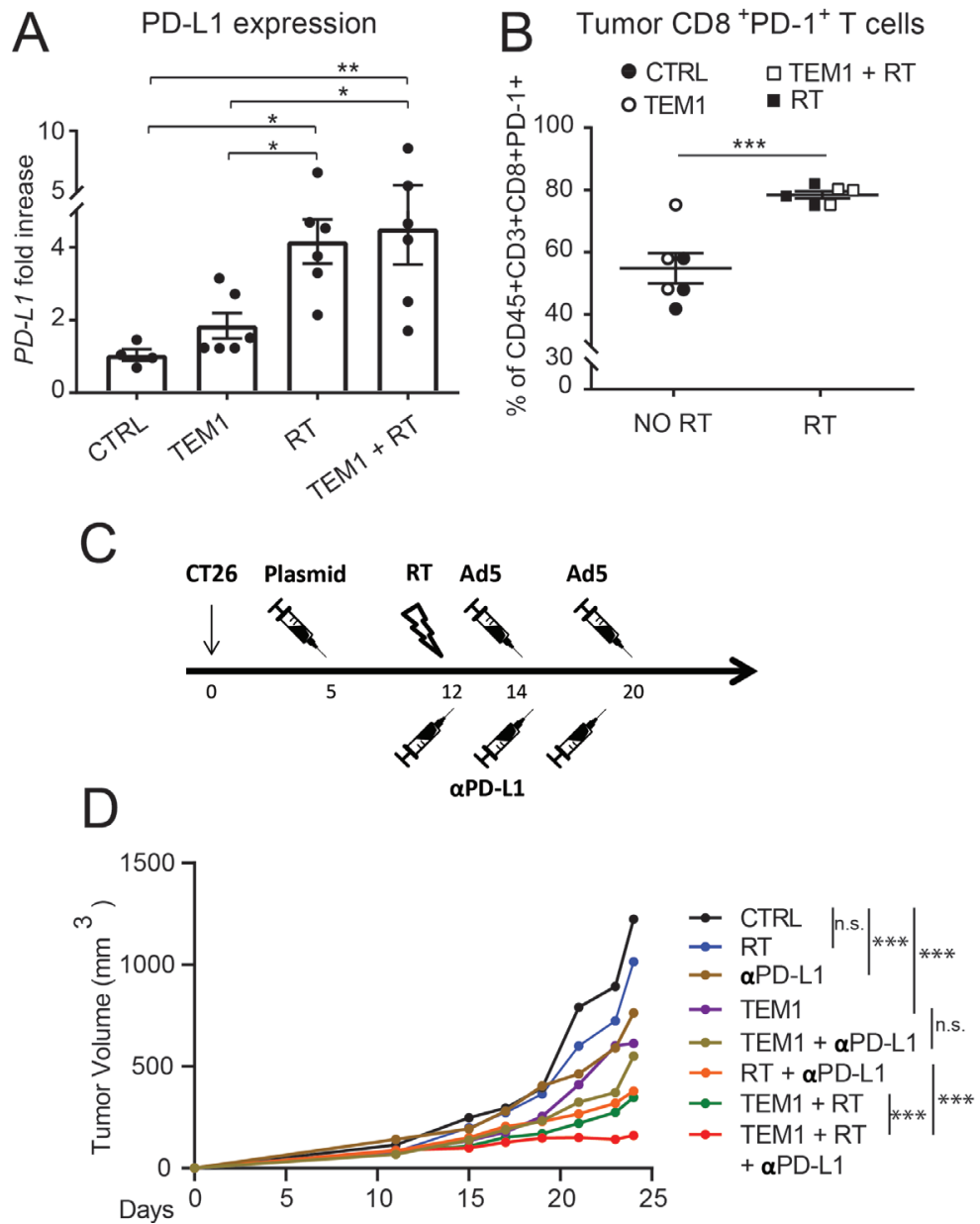

E
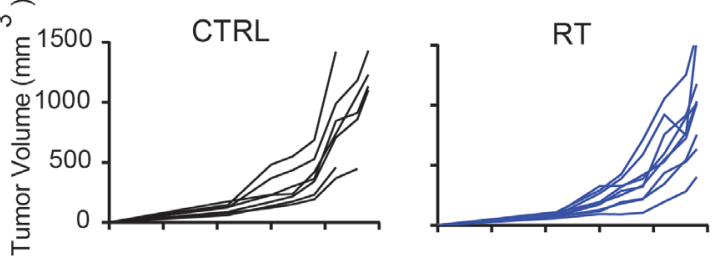

?
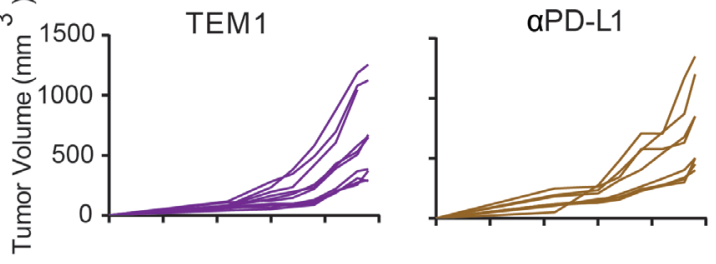

m
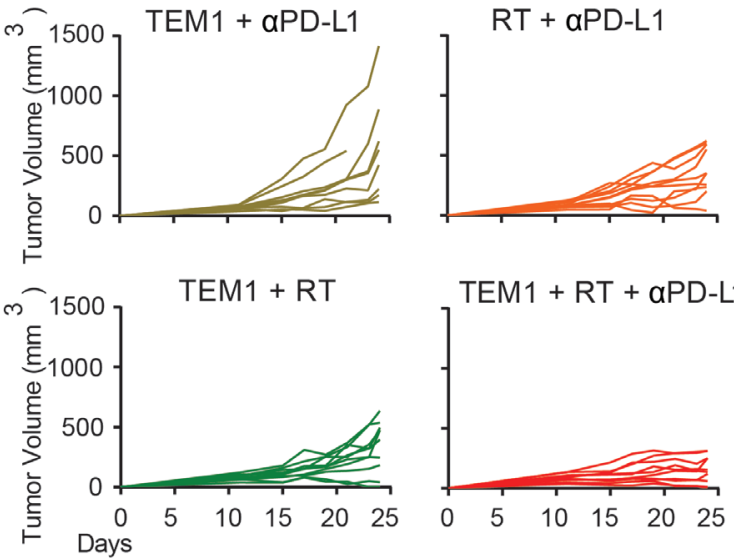

Figure 6 Inhibition of PD-1/PD-L1 axis enhances the antitumor effects of dual therapy. CT26-bearing mice were sacrificed, and tumors were analyzed by real time-PCR (A) and by flow cytometry (B). Real time-PCR shows fold-change expression of PD-L1. All measures were made in triplicates. (B) Flow cytometry analysis performed on tumor-single cell suspension shows proportion of $\mathrm{PD}-1^{+} \mathrm{CD} 8^{+} \mathrm{T}$ cells within the primary tumor. Each dot represents a mouse. Tukey's multiple comparison tests were performed ( $A$, CTRL vs RT $p=0.03$; TEM1 vs RT $p=0.05$; CTRL vs TEM1+RT $p=0.009$; TEM1 vs TEM1+RT $p=0.04$. $B, p<0.001$ ). (C) Schematic representation of the therapeutic protocol. (D and E) BALB/c mice were injected in both flanks with CT26 at 2day interval, and then RT was performed only in the primary tumor. Means (D) of abscopal tumor growth or individual tumor sizes (E) are shown and data are representative of one independent experiment out of three $(n=7-10)$. Differences in tumor volume were evaluated with mixed model (CTRL vs RT $p=0.075$; CTRL vs TEM1 $p<0.001$; CTRL vs $\alpha$ PD-L1 $p<0.001 ; \alpha P D-L 1$

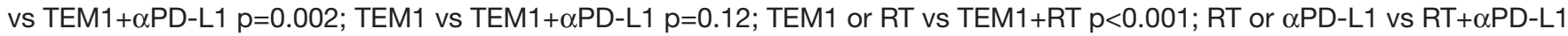

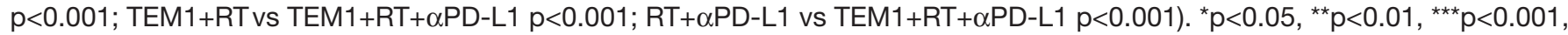
n.s. non-significant. PD1, programmed cell death protein 1; PD-L1, programmed death-ligand 1; RT, radiation therapy; TEM1, tumor endothelial marker 1; $\alpha$ PD-L1, anti-PD-L1 antibody.

RT, and $\alpha$ PD-L1 resulted in $83.3 \%$ of complete responses (figure 7A) and significantly improved long-term survival compared with RT $+\alpha$ PD-L1 therapy while only trending toward longer survival compared with TEM1+RT combo. Finally, although showing a similar trend as CT26, triple combination therapy in the TC1 model did not significantly improve mice outcome compared with TEM1+RT combo (online supplemental figure S9). Cumulatively, we observed that mice receiving radiation significantly enhanced PD-1/PD-L1 expression within their tumors. Addition of $\alpha \mathrm{PD}-\mathrm{L} 1$ to TEM1 vaccination+RT significantly increases antitumor effects in the CT26 model.

\section{Inhibition of PD-1/PD-L1 axis with heterologous TEM1 vaccination and $R T$ augments gp70-specific responses while reducing the TEM1-specific response}

To characterize the mechanism(s) underlying the enhanced systemic antitumor effect achieved with the triple combination treatment, we examined the frequency of $\mathrm{T}$ cell immune responses against TEM1 and gp70 antigens. As reported previously, combining RT with the TEM1 vaccine led to a significant increase in the frequency of TEM1-specific $\mathrm{T}$ cells in the spleen (figures 4A and 7B) compared with just vaccine. Unexpectedly, blocking PD-L1 during the dual treatment (TEM1+RT+ $\alpha$ PD-L1) reduced the TEM1-specific response (figure 7B) relative to dual treatment. In the same mice, we observed a significant increase in the peripheral anti-gp70 response, as 
A

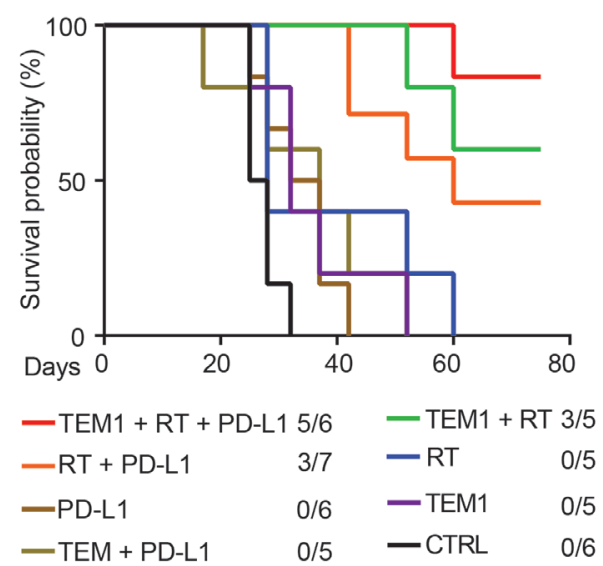

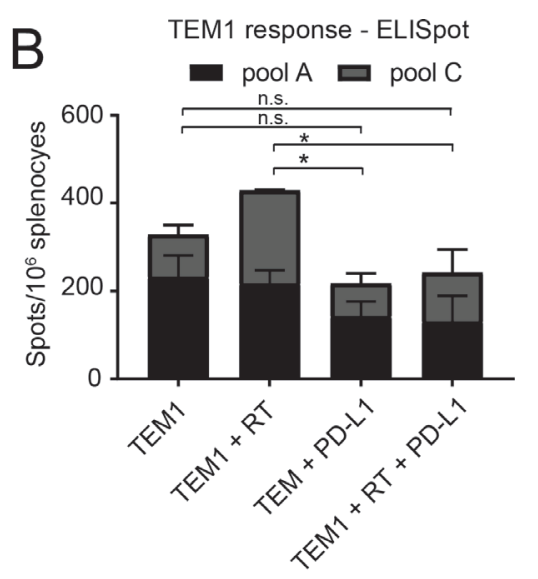
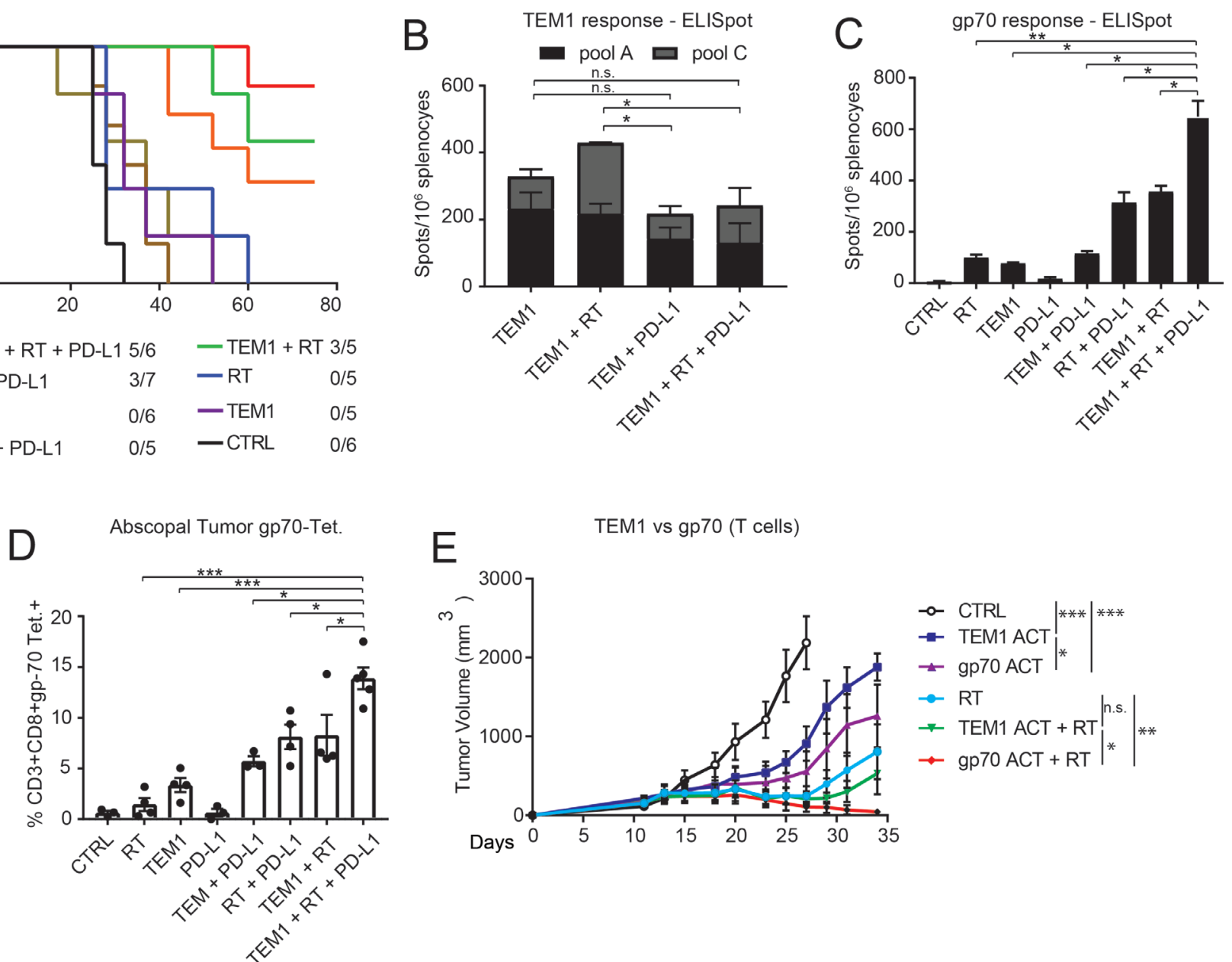

Figure 7 Inhibition of PD-1/PD-L1 axis with heterologous TEM1 vaccination and RT augments gp70-specific responses while reducing the TEM1-specific response (A) BALB/c mice were challenged in the lower back with the CT26 tumor and treated. Mice were considered expired when tumor reached $2000 \mathrm{~mm}^{3}$. The Kaplan-Meier plots shows survival probability of mice in each group. Data are representative of one independent experiment out of two $(n=5-7)$. Statistical significance were calculated with log-rank (Mantel-Cox) test (CTRL vs RT $p=0.061$; CTRL vs $\alpha P D-L 1 p=0.041$; CTRL vs TEM1 $p=0.0412 ; R T$ vs

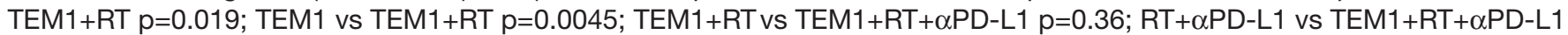
$\mathrm{p}=0.011$ ). (B and $\mathrm{C}) \mathrm{CT} 26$-bearing mice were sacrificed to analyze spleen and tumors. ELISpot assay performed on splenocytes stimulated with TEM1 peptide library $(B)$ or $\mathrm{AH} 1$ peptide $(\mathrm{C})$ shows frequency of peripheral immune response after each therapy. (D) Quantification by flow cytometry shows percentage of gp70-specific T cells (gp70-Tet.) infiltrating the abscopal tumor. Percentage of total CD8 ${ }^{+} \mathrm{T}$ cells is shown. Tukey's multiple comparison tests were performed (B, TEM1 vs TEM1+RT+ $\alpha \mathrm{PD}-\mathrm{L} 1$

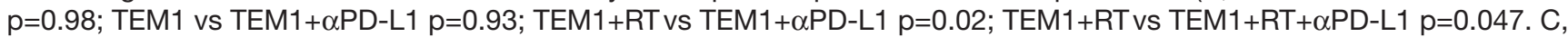

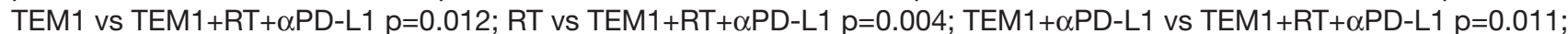

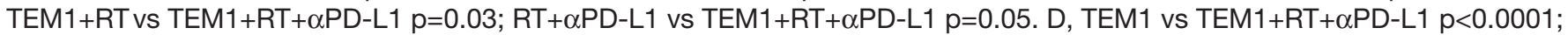

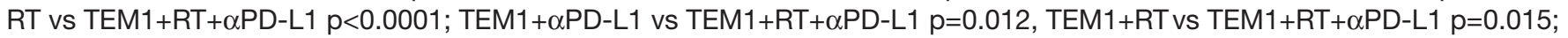
$R T+\alpha P D-L 1$ vs TEM1+RT+ $\alpha P D-L 1 \quad p=0.012$ ). Means $\pm S E M$ are shown. Each dot represents a mouse. (E) CT26 tumor-bearing mice were adoptively transferred (ACT) with TEM1-specific or gp70-specific T cells isolated from vaccinated mice. In some groups, mice were also treated with RT at day 12 (1 hour before ACT). Differences in tumor volume were evaluated with twoway analysis of variance (ANOVA) test (CTRL vs TEM1ACT or gp70ACT $p<0.001$; TEM1ACT vs gp70ACT $p=0.02$; RT vs TEM1ACT+RT $p=0.183$; RT vs gp70ACT+RT $p=0.002$; TEM1ACT+RT vs gp70ACT+RT $p=0.015)$. data are representative of one independent experiment out of two $(n=5-6)$. Means \pm SEM are shown. ${ }^{*} p<0.05,{ }^{\star \star} p<0.01$, ${ }^{\star *} p<0.001$, n.s. non-significant. PD1, programmed cell death protein 1; PD-L1, programmed death-ligand 1; RT, radiation therapy; TEM1, tumor endothelial marker 1; $\alpha P D-L 1$, anti-PD-L1 antibody.

measured by ELISpot (figure 7C) and tetramer staining (online supplemental figure S10A), suggesting that $\alpha$ PD-L1 treatment skewed $\mathrm{T}$ cell receptor specificity toward the gp70 specific viral TAA. This increased peripheral immune response correlated with improved gp70specific $\mathrm{T}$ cell infiltration within abscopal tumors of mice treated with the triple combination therapy (figure 7D), compared with mice treated with the dual therapies.

To assess the potency and expansion of TEM1-specific and gp70-specific $\mathrm{T}$ cells in a therapeutic setting, we performed adoptive cell transfer (ACT) experiments in which we transferred the same number of 
antigen-reactive T cells (both TEM1 and gp70) into CT26 tumor-bearing mice. ${ }^{43}$ Reactive T cells were generated on vaccination of healthy non-tumor-bearing mice using the Tem1-TT and the IgE-gp70-TT plasmid-DNA vaccines. To measure levels of antigen-reactive $T$ cells, we performed IFN- $\gamma$ intracellular cytokine staining (ICS) on blood (online supplemental figure S10B) of vaccinated mice and showed that efficacy of the gp70 vaccine $(4.2 \%$ IFN- $\gamma^{+} \mathrm{T}$ cells) was approximately $30 \%$ higher than the TEM1 vaccination (3.2\% IFN- $\gamma^{+} \mathrm{T}$ cells). Therefore, to transfer the same number of antigen-specific $\mathrm{T}$ cells, we adoptively transferred $30 \%$ more TEM1-specific T cells $\left(6.5 \times 10^{6}\right)$ than gp70 $\mathrm{T}$ cells $\left(5 \times 10^{6}\right)$. As shown in the ACT experiment in figure $7 \mathrm{E}$ and online supplemental figure S11, adoptive transfer of gp70-specific T cells was more effective than transfer of TEM1-specific T cells both with and without radiation. In summary, blocking PD-1/ PD-L1 signaling during TEM1 vaccination and RT dual treatment promotes expansion of gp70-specific $\mathrm{T}$ cells while simultaneously dampening the vaccine-specific (anti-TEM1) response.

\section{DISCUSSION}

Although therapeutic vaccination with the TEM1 plasmid-DNA resulted in delayed tumor progression, reduced tumor MVD, lower blood perfusion of the tumor, and increased tumor hypoxia in three different mouse tumor models, TEM1 plasmid-DNA did not completely eradicate tumors. ${ }^{13}$ Several strategies to improve DNA-based vaccination efficacy have been investigated. ${ }^{11} 1718$ DNA prime followed by boosting with viral vectors has been used to enhance immune responses against malaria, ${ }^{23}$ viruses, ${ }^{2425}$ and cancers. ${ }^{26-29}$ Heterologous injections with plasmid-DNA vaccine followed by adenoviral (Ad5) vaccine, both encoding guanylyl cyclase C (GUCY2C), have been shown to effectively control CT26 tumor progression. ${ }^{44}$ Similarly, a protocol consisting of priming with plasmid-DNA vaccine followed by boost injections with an adenoviral vaccine, both expressing a truncated human epidermal growth factor receptor 2 (HER2), has been shown to elicit Her-2/neu-specific humoral and cellular immune responses without causing severe adverse effects in monkeys. ${ }^{45}$ Based on those encouraging results, a phase I study (NCT01895491) was conducted in patients with stage III-IV metastatic breast cancer. In line with the preclinical data, HER2-specific cell-mediated and humoral responses was reported in some patients leading to objective responses without serious adverse events. ${ }^{26}$ Based on our previous findings that heterologous prime/ boost vaccination can enhance vaccine efficacy, ${ }^{20}{ }^{22}$ we cloned the TEM1 insert into a replication-deficient Ad5 adenoviral vector and then tested the novel vaccination protocol. We found that priming with the TEM1 plasmid-DNA followed by two boosts with TEM1 Ad5 bolstered the immune response against TEM1-specific peptides and improved DNA vaccine antitumor effects.
Over the last decade it has become increasing clear that cancer treatment modalities have improved, although incrementally, by combination therapy. Owing to the ability of RT to modulate the TME, we carried out in vivo experiments to determine whether localized RT of TC1 and CT26 tumors can enhance the antitumor effect of the heterologous TEM1 vaccine. We report the combination of RT and TEM1 vaccination results in significant improvement in tumor control and increased survival compared with single therapy. Combination therapy was effective if radiation was delivered early (ie, Plasmid-RTAd5-Ad5), rather than late (ie, Plasmid-Ad5-Ad5-RT) in both tumor models. In the "early" protocol, RT is given when the anti-TEM1 immune response is primed but not expanded, and hence the tumor is not fully infiltrated. In this case, RT would induce TME remodeling and spark cross priming but also likely affect the anti-TEM1 T cells in the vicinity of the tumor. The sequential adenovirus boost would then promote expansion of TEM1 T cells, replenishing the depleted cells and promoting tumor $\mathrm{T}$ cells infiltration. The difference in efficacy between the two protocol is also likely explained by the observation that large tumors are more immunosuppressive than smaller tumors and hence more resistant to immunotherapy. ${ }^{46}$

One major mechanism underlying the enhanced antitumor effect of combining RT and TEM1 vaccination includes the vascular targeting property that both individual therapies possess. Antiangiogenic and vasculardisrupting therapeutics have been developed as a mode of starving the tumor of oxygen and nutrients. ${ }^{47}$ Protocols that aim to induce adaptive $T$ cell responses specific to the tumor vasculature have also been studied, ${ }^{48} 49$ reporting only modest efficacy when used as monotherapy. ${ }^{50}$ More recently, $\mathrm{T}$ cells engineered with a novel nanobodybased chimeric antigen receptor against VEGFR2 have been reported but safety was not adequately addressed. ${ }^{51}$ Another mechanism attributed to vascular disruption therapy is vascular normalization. Restoring blood perfusion by eliminating the less mature, stable vessels and stabilizing remaining vessels has been shown to enhance drug delivery. ${ }^{53}$ Accordingly, we cannot dismiss the possibility that TEM1 vaccine-elicited $\mathrm{T}$ cells may also target the non-coordinated or poorly formed vasculature therefore decreasing intratumoral pressure and favoring T cell infiltration. ${ }^{54}$

Based on our results that TEM1 plasmid vaccination impacts tumor endothelium, ${ }^{13}$ and in concert with the findings that high-dose radiation can disrupt the tumor vasculature, ${ }^{9}$ we examined the effects of dual treatment with heterologous TEM1 vaccine and RT to assess potential cooperativity from each vascular-disrupting modality. Since radiation can augment surface expression of MHCI molecules on cancer cells ${ }^{55}$ we analyzed RT-mediated effects on EC and observed a dose-dependent increase in MHCI surface expression. Coculture of irradiated H5V $\mathrm{EC}$ with TEM1-specific $\mathrm{T}$ cells increased $\mathrm{T}$ cell activation, suggesting that RT promotes immune recognition of tumor endothelium. We observed similar findings in vivo 
as RT-treated tumor showed increased MHCI and TEM1 expression on tumor-associated EC. In parallel findings, RT also increases vaccination efficacy leading to greater peripheral expansion of anti-TEM1 T cells, which can subsequently target the "immunologically recognized" endothelium via upregulation of MHCI and TEM1. This unforeseen partnership between the two therapies leads to synergistic damage of the tumor vasculature and augmented control of primary tumor growth. While it has been previously reported that radiation can enhance MHCI expression on and immune recognition of tumor cells, ${ }^{5}$ to our knowledge, we are the first to report and characterize similar effects on the endothelium of the tumor and this MHCI modulation to be sufficient to increase recognition by $\mathrm{T}$ cells.

Effective management of both local and metastatic malignant sites is a fundamental challenge in cancer treatment. In recent years, the impact of hypofractionated radiotherapy on the antitumor immune response has been intensively studied ${ }^{5657}$ Based on these findings, we asked whether treatment with TEM1 vaccination and RT would result in an abscopal effect, and indeed found a significant antitumor effect on the non-irradiated tumor when the dual therapy was administered. Varying radiation doses and delivery schedules have been investigated to determine an optimized antitumor effect. Results from a recent study suggest that doses greater than 12-18 Gy of RT may attenuate the abscopal effect by degrading DNA that accumulates in the cytosol after irradiation. ${ }^{58}$ Perhaps due to the different models tested, we did observe a robust systemic response in mice that received a single $21 \mathrm{~Gy}$ dose of RT together with TEM1 vaccination. Possible mechanisms underlying the abscopal effects induced by combining irradiation with immunotherapy include improved TAA cross-presentation ${ }^{2}$ and increased number of tumor-infiltrating lymphocytes. ${ }^{59-62}$ We consistently observed that dual treatment with TEM1 vaccination and RT increased epitope spreading toward TAA (ie, AH1 and E7) and enhanced $\mathrm{CD}^{+}$lymphocyte infiltration, specifically TAA-restricted cells, within the abscopal tumor in both CT26 and TC1 models. Another RT-mediated effect that may occur within the TME is increased activation of DC, which can in turn promote effective TAA crosspresentation. ${ }^{1}$ To test for this phenomenon, we examined irradiated tumors and draining LNs and found that dual treatment resulted in increased CD11 $\mathrm{c}^{+}$cells-which include both DC and macrophage-activation compared with the untreated group. In subsequent experiments, we demonstrated that $\mathrm{CD} 11 \mathrm{c}^{+}$are specifically required for the functional stimulation of gp70-specific T cells, linking TME modulation with an increased adaptive immune response.

PD-1/PD-L1 ICI has achieved great clinical success, nonetheless, a large proportion of patients (up to $80 \%$ ), including those with positive PD-L1/PD-1 expression, do not respond to PD-1/PD-L1 blockade. ${ }^{63}$ Previous studies have also shown that RT can hinder treatment efforts by upregulating proteins, such as PD-L1, that contribute to immunosuppression. ${ }^{41}$ Moreover, "chronic" IFN- $\gamma$ signaling can augment PD-1/PD-L1 expression within tumors. ${ }^{64}$ In our model, we found that PD-1, PD-L1 and IFN- $\gamma$ were all upregulated in response to the combination therapy in CT26 tumors. Based on these findings, we hypothesized that we could further enhance the effects of the dual treatment by also blocking the PD-1/PD-L1 axis via administration of an $\alpha$ PD-L1. Indeed, in the CT26 model, triple combination therapy with heterologous TEM1 vaccine, RT and $\alpha$ PD-L1 resulted in a significantly stronger abscopal effect and tumor control than any of the dual treatment regimens. We tested the triple combination also in the TC1 model and, although we observed a similar trend as for the CT26 model, addition of ICI to the TEM1+RT combination did not statistically improved outcome. This differential therapeutic effect of the triple combination between CT26 and TC1 tumor models can be explained by the previously published inherent differences in the immunogenicity of the TEM1 vaccine between the mouse strains (BALB/c vs C57BL6) ${ }^{13}$ as well as the tumor models (CT26 vs TC1) ${ }^{65-68}$ Although similar combinations have been previously investigated, this is the first report to show direct tumor vasculature targeting by vaccine in combination with radiation and ICI. We also report an unexpected finding that blocking PD-1/PD-L1 interaction during the dual therapy vastly enhances cross-presentation of the dominant gp70 viralantigen while paradoxically suppressing vaccine-induced response against the self TEM1 antigen. This suggests that TEM1 vaccination and vascular targeting is required for the initial elicitation of potent TAA cross-priming but is less relevant in the subsequent antitumor effects of the combination. Our finding is in line with a previous study showing that blocking PD-1/PD-L1 signaling during administration of a DC-vaccine pulsed with multiple peptides promotes expansion of $\mathrm{T}$ cells against the dominant antigen while other T cells clones were suppressed. ${ }^{69}$ However, to the best of our knowledge, this is the first report to demonstrate that anti-PD-L1 treatment can suppress a vaccine-induced immune response while enhancing epitope spreading towards a TAA.

Taken together, our data supports a model (online supplemental figure S12) in which the addition of radiation with TEM1 vaccination augments MHCI expression on EC, enhances their immune recognition by anti-TEM1 $\mathrm{T}$ cells and results in increased endothelial and tumor cell death. Dead cells are subsequently scavenged by RT-activated DC/macrophage that process and cross-present additional TAA to T cells. This secondary cross-priming event promotes expansion of TAA-specific $\mathrm{T}$ cells (epitope spreading), which acts in partnership with the anti-TEM1 immune response to eradicate larger numbers of tumor and EC, resulting in improved control of primary and abscopal distant tumors. Addition of antiPD-L1 to the dual therapy enhances the gp70-specific response and improves the antitumor effect.

Collectively, the data presented here clearly demonstrates the importance of a rational combinational 
approach for cancer therapy and the opportunity to obtain sequential additive effects that maximize potency of the anti-TEM1 therapy. Our findings provide the preclinical rationale that combination therapy with heterologous TEM1 vaccination and RT is a rationale add-on therapy to established PD-1/PD-L1 blockade therapy, and as a potential approach as dual therapy in its own right, and holds promise as a novel antitumor combination approach with potential for translation into the clinic.

\section{MATERIALS AND METHODS}

\section{Animal studies and cell lines}

Six-week-old to 8-week-old C57BL/6 $\left(\mathrm{H}-2^{\mathrm{b}}\right)$ and BALB/c $\left(\mathrm{H}-2^{\mathrm{d}}\right)$ mice were purchased from Jackson Laboratory (Bar Harbor, Maine). TC1 (H-2 $)$, CT26 (H-2 $\left.{ }^{\mathrm{d}}\right)$, and $\mathrm{H} 5 \mathrm{~V}\left(\mathrm{H}-2^{\mathrm{b}}\right.$ ) cells were cultured in RPMI 1640 (Cellgro; \#10-104-CV) medium supplemented with $2 \mathrm{mM}$ L-glutamine (Gibco, \#35 050-061) and $150 \mathrm{U} / \mathrm{mL}$ streptomycin plus $200 \mathrm{U} / \mathrm{mL}$ penicillin (Cellgro; \#30-0010 CI) and 10\% heat-inactivated FBS (Life Technologies, \#16000044). All cell lines were obtained from ATCC and used within 15-20 passages. The CT26 MHCI-deficient cell line was obtained by knockdown of the beta- 2 microglobulin gene (B2M) using "TRC lentiviral shRNA technology" (Dharmacon; RMM4534-EG12010). Puromycin selection $(10 \mu \mathrm{g} / \mathrm{mL}$; InvivoGen; \#ant-pr-1) was used to select for cells containing the plasmid, and cell sorting was used to generate single cell population.

\section{DNA and adenovirus vectors}

The TEM1 plasmid-DNA was generated by fusing the fulllength mouse Tem 1 cDNA with a cDNA corresponding to the N-terminal domain of fragment C of TT (865-1120). The TT fragment DNA was introduced at the 3 ' end of the Tem1 coding sequence, generating the sequence Tem1TT, which was then inserted into a pVAX plasmid (ThermoFisher Scientific; \#V26020). The Tem1-TT cDNA was codon usage-optimized for mouse and synthesized by oligonucleotide assembly (GeneArt; Life Technologies). The gp70 plasmid-DNA was generated by fusing the gp70 cDNA with the cDNA of the N-terminal domain of fragment $\mathrm{C}$ of TT. The IgE leader sequence (aa 1-18) preceded the gp70 sequence. The resulting IgE-gp70-TT sequence was codon-optimized, synthesized and cloned into a pVAX vector by GeneArt. Gp-70-specific T cells were used for antigen recognition experiments and ACT therapy. The TEM1 adenoviral vector Ad5 (Human Type 5 (dE1/E3)) was generated by Vector Biolabs (Malvern, $\mathrm{PA}$ ) via subcloning the $3.1 \mathrm{~kb}$ insert (Tem1-TT) using EcoRI/PspOMI into pD2-MCS shuttle vector. The virus was packaged into the 293 cells. All constructs were routinely sequenced by the DNA sequencing core facility at University of Pennsylvania.

\section{Vaccination procedures}

Plasmid-DNA immunization was performed as described previously. ${ }^{13}$ Briefly, $50 \mu \mathrm{g}$ of plasmid in phosphate-buffered saline(PBS) was injected intramuscularly and electroporation was performed (2 pulses at $100 \mathrm{mV}$ for $200 \mathrm{~ms}$ ) immediately after injection. For adenovirus injections, $1 \times 10^{9} \mathrm{pfu} /$ mouse were injected intramuscularly in $50 \mu \mathrm{L}$ PBS. Heterologous vaccination consisted of priming with the plasmid-DNA followed by two adenovirous injections. All the vaccination modalities (plasmid alone, adenovirus alone, and heterologous vaccination) consisted of three injections performed at weekly intervals.

\section{Synthetic peptides}

The mouse TEM1 library was synthesized by Mimotopes. The 15-mer peptides were dissolved in dimethylsulfoxide (DMSO) at $20 \mu \mathrm{g} / \mu \mathrm{L}$ and were divided into pools A-D, with approximately 38 peptides each (pool D contained fewer peptides). Pools and individual peptides were used at $1 \mu \mathrm{g} / \mathrm{mL}$ for ELISpot. Once immunoreactive pools were identified, peptides were tested individually to identify the immunodominant peptide/s. The gp70 peptide (Anaspec, \#AS-64798) was re-suspended in DMSO and used at $1 \mu \mathrm{g} / \mathrm{mL}$.

\section{Irradiation}

In vitro cultures of CT26, CT26-MHCI ${ }^{-}$, and $\mathrm{H} 5 \mathrm{~V}$ cell lines were irradiated with 5, 10, or $20 \mathrm{~Gy}$ using XRAD320iX followed by different assays (Flow cytometry, real time-PCR, and coculture). In vivo tumors were irradiated with $21 \mathrm{~Gy}$ single dose at 10 or 12 day (Early $R T$ ) or at 19 or 21 day (Late RT) (for TC1 or CT26 cell lines, respectively) after tumor inoculation when tumor volumes were between 100 and $250 \mathrm{~mm}^{3}$ (TC1) or 200 and $350 \mathrm{~mm}^{3}$ (CT26) in the early RT and about $1400 \mathrm{~mm}^{3}$ (TC1) and about $1600 \mathrm{~mm}^{3}$ (CT26) in the late RT using an XRAD320iX, an X-ray system capable of delivering a precise radiation dosage to small animals such as mice. The process included lead shielding of the non-irradiated area to protect the mouse from the irradiation. Irradiation of tumor draining LNs was avoided in order to allow T cell priming. The Department of Radiation Oncology houses an X-Rad 320IX Biological X-Ray Irradiator (Precision X-Ray, North Branford, Connecticut, USA) in the Smilow Center for Translational Research. The irradiator is calibrated for absolute dose using the AAPM TG-61 (AAPM protocol for 40-300 kV $\mathrm{X}$-ray beam dosimetry in radiotherapy and radiobiology, Medical Physics, 28, ${ }^{6}$ June 2001) protocol for 138 kV X-ray beam dosimetry. The calibrations are performed with a 0.6 cc volume Exradin Model A12 (Standard Imaging, Middleton, WI, USA) Farmer chamber and paired Welhoffer Dose 1 (IBA Dosimetry, Bartlett, Tennessee, USA) electrometer, both with National Institute of Standards and Technology (NIST) traceable accredited dosimetry calibration laboratory (ADCL) calibration certificates. Regular film and OSLD measurements are performed to monitor irradiator output.

\section{IFN- $\gamma$ ELISpot}

Ninety-six-well MAIP plates (Millipore; \#N4510) were coated overnight with a 1:400 dilution of rat antimouse 
IFN- $\gamma$ (BD Biosciences; clone R4-6A2, \#551216) in sterile PBS. Splenocytes were plated at $0.1-1 \times 10^{6}$ cells/well and incubated overnight at $37^{\circ} \mathrm{C}$ with $1 \mu \mathrm{g} / \mathrm{mL}$ peptides. Following the incubation, the plates were washed with PBS and $0.05 \%$ Tween-20 (Bio-Rad; \#170-6531) and incubated with antimouse biotin-conjugated anti-IFN- $\gamma$ antibody (BD Biosciences; clone R4-6A2, \#551506). After washing the plate, streptavidin-alkaline phosphatase conjugate (BD Biosciences; \#554065) was then added for $30 \mathrm{~min}$. Plates were developed by adding nitroblue tetrazolium/5bromo-4-chloro-3-indolyl phosphate (Pierce), and spots were then counted using an automated ELISpot reader (Autoimmun Diagnostika GmbH).

\section{Coculture assay and immune recognition}

$2 \times 10^{4}$ CT26 or CT26 MHCI $^{-}$and $5 \times 10^{4} \mathrm{H} 5 \mathrm{~V}$ were irradiated and 6 hours later cells were cocultured overnight with $1 \times 10^{5}$ of gp70-specific or TEM1-specific $\mathrm{CD}^{+} \mathrm{T}$ cells in an IFN- $\gamma$ ELISpot plate. $\mathrm{CD}^{+} \mathrm{T}$ cells were isolated from splenocytes of vaccinated mice using Pan T cell Isolation Kit II mouse (Miltenyi Biotech, \#130-095-130). Isolation of DC/macrophage from the draining LNs was achieved by cell sorting of $\mathrm{CD} 11 \mathrm{c}^{+}$cells performed at the Flow Cytometry and Cell Sorting Facility at the University of Pennsylvania. A total of $1 \times 10^{4} \mathrm{DC}$ from treated mice were cocultured overnight with $1 \times 10^{5}$ of gp70-specific $\mathrm{CD}^{+} \mathrm{T}$ cells in an IFN- $\gamma$ ELISpot plate. A final concentration of $1 \mu \mathrm{g} / \mathrm{mL}$ of gp70 peptide was added in the coculture.

\section{Flow cytometry}

Tumor cell suspensions were stained, and single cell analysis was performed by flow cytometry using 8-parameter flow cytometry on a FACSCanto (BD). To identify positive and negative populations, gates were set based on pilot experiments and on our experience running similar experiments. Fluorescence minus one, isotype controls and/or use of negative samples were used to determine gate boundaries. Additionally, antibody titration was used to optimize antibody panels. Immune phenotyping of mouse samples was performed following staining with: anti-CD45 (eBioscience; clone 30-F11, \#48-0451-82), antiCD11b (eBioscience; clone M1/70, \#12-0112-82), antiCD11c (eBioscience; clone N418, \#69-0114-82), anti-Gr-1 (eBioscience; clone RB6-8C5, \#25-5931-82), anti-MHCII (Biolegend; clone M5/114.15.2, \#107606), anti-CD40 (Biolegend; clone 3/23, \#124622), anti-MHCI (H-2Kd) (eBioscience; clone SF1.1.1.1, \#17-5957-82), anti-MHCI (H-2Kb) (Biolegend; clone AF6-88.5, \#116518), and antiPD-L1 (Biolegend; clone 10F.962, \#124312), anti-CD103 (eBioscience; clone 2E7, \#17-1031-82), anti-CD30 (eBioscience; clone 390,\#11-0311-82), anti-CD8a (eBioscience; clone 53-6.7, \#11-0081-85), anti-CD3 (eBioscience; clone 17A2, \#69-0032-82), anti-CD4 (eBioscience, clone GK 1.5, \#25-0041-82), anti-PD-1 (eBioscience; clone RMPI30, \#46-9981-82), anti-IFN $\gamma$ (eBioscience; clone XMG1.2, \#48-7311-82), and H-2Ld MuLV gp70 tetramer (MBL, \#TS-M521-1). For all experiments, a LIVE/DEAD cell stain kit (Invitrogen, \#L34966) was used.

\section{Real time-PCR}

The relative quantification ${ }^{70}$ of the expression levels of selected genes was carried out by real time-PCR using an ABI PRISM Viia7 (Applied Biosystems). Total RNA from tissues was extracted using Trizol reagent (Invitrogen) according to the manufacturer's instructions. The purity of the RNA samples was determined by visualization of intact $18 \mathrm{~S}$ and 28S RNA bands in agarose gel electrophoresis. Two micrograms of RNA were used for cDNA synthesis using high-capacity cDNA reverse transcription kit (Applied Biosystem, \#4368814). Fifty nanograms of cDNA were used in each real time-PCR reaction run. The following TaqMan gene expression assays were used to quantify expression levels of mGAPDH (Mm99999915 g1), mTEM1 (Mm00547485_s1), mPDL1 (Mm00452054_ $\mathrm{m} 1)$, and mIFN- $\gamma$ (Mm01168134_m1).

\section{Tumor challenge}

C57BL/6 and BALB/c mice were injected subcutaneously on the lower back (one-tumor model) or on both flanks (two-tumor model) with $2.5 \times 10^{5}$ cells/mouse (CT26) or with $1 \times 10^{5}$ cells/mouse (TC1). The cells were injected with matrigel (Corning, \#356231) at a 1:1 ratio. The injection volume was $100 \mu \mathrm{L} /$ mouse. In the two-tumor model, the irradiated tumor was injected first and 2 days after the abscopal (non-irradiated) tumor was injected in the contralateral flank. All cell lines were propagated in 5\% $\mathrm{CO}_{2}$ at $37^{\circ} \mathrm{C}$ and checked for mycoplasma contamination before tumor challenge.

\section{Immunohistochemical and immunoflourescence tumor analyses}

Tumors were embedded in OCT medium or immediately frozen in dry ice. Sections $(10 \mu \mathrm{m}$ thick) were stained for mouse CD3 (Abcam; Rb mAb to CD3 epsilon, \#ab215212). Images of the slides were taken using an Olympus PROVIS microscope. For immunofluorescence, OCT-embedded tumors were sectioned as $10 \mu \mathrm{m}$ thick slices and stained for mouse CD31 (Abcam; Rb pAb to CD31, \#ab28364). As secondary antibody, Alexa Fluor 488 goat anti-rabbit IgG (Invitrogen; \#A11008) was used with Hoechst 33342 (Invitrogen; \#H3570) as a counterstain. Images of the slides were taken on an Observer.Z1 Zeiss microscope using Zeiss Zen Pro (Zeiss). Two investigators performed a blind assessment of the sections.

\section{Ultrasound analysis of tumor vasculature}

Lipid-coated microspheres filled with octafluoropropane gas (Definity, Lantheus Medical Imaging) were used as an ultrasound contrast agent with power Doppler imaging to visualize the regions of perfusion in the tumor. In brief, CT26 tumor-bearing mice (tumor volume, $\sim 600 \mathrm{~mm}^{3}$ ) were anesthetized and injected intravenous with $0.02 \mathrm{~mL}$ of definity. Power Doppler imaging was performed using the MS250 transducer which scanned at a frequency of $18 \mathrm{mHz}$ (Visualsonics, Toronto, Canada). Power Doppler images were acquired at a frame rate of $10 \mathrm{~Hz}$ to minimize microbubble destruction by the imaging ultrasound 
pulses. The Doppler signal from the inflowing contrast agent was visible in the images as a color superimposed on the grayscale image of the tumor. The power Doppler images at peak enhancement were analyzed to determine percentage area of the tumor with flow (percentage perfused area). The perfused tumor area for each treated group was calculated as the ratio of the area resolution by the contrast agent to the total tumor area in a single plane. Color-weighted fractional area of the colored pixels within the region of interest was measured as described previously. The color of each pixel in the contrastenhanced power Doppler image measures the fractional volume of the contrast flowing through the pixel, and the color-weighted fractional area (product of color level and fraction area covered by colored pixels) measures the contrast volume per unit area of tumor (blood flux).

\section{In vivo anti-PD-L1 antibody treatment}

The mice received three doses (every 3 days) of $200 \mu \mathrm{g} /$ mouse of anti-PD-L1 (BioXcell; clone 10F.9G2, BE0101) blocking antibody by intraperitoneal injection.

\section{Adoptive transfer of cells}

ACT were performed as described. ${ }^{71}$ Tumor-free mice were vaccinated three times with either TEM1 or gp70 vaccine, and IFN- $\gamma$ ICS was performed 1 week after the last immunization to test vaccine efficacy. One day after ICS, CD3 ${ }^{+} \mathrm{T}$ cells were magnetically isolated from the spleens of vaccinated mice and injected itravenous $\left(6.5 \times 10^{6}\right.$ TEM1-specific T cells and $5 \times 10^{6}$ gp70-specific $\mathrm{T}$ cells) into tumor-bearing mice (challenged 12 days before ACT) that had been sublethally irradiated (4Gy of total body irradiation) to achieve lymph depletion 2 days before ACT. Mice were given a single $21 \mathrm{~Gy}$ dose of radiation 1 hour before ACT. Tumor volume at irradiation has mean volume of about $300 \mathrm{~mm}^{3}$.

\section{Statistics}

For comparisons of more than two groups, we used Tukey's multiple comparison tests. For all other comparisons, two-tailed Student's t-tests using a pooled estimate of the variance were used. For mouse experiments, we used a two-way analysis of variance (ANOVA) tests or a mixed mode based on the type of dataset. When some values were missing, data are analyzed by fitting a mixed model, rather than by repeated measures used by ANOVA (which cannot handle missing values). Mice group sizes were chosen based on pilot experiments and on our experience carrying out similar experiments. Mouse numbers and the specific statistical tests used are specified in each figure legend. $* \mathrm{p}<0.05, * * \mathrm{p}<0.01, * * * \mathrm{p}<0.001$, n.s. (non-significant).

\section{Author affiliations}

${ }^{1}$ Department of Radiation Oncology, Perelman School of Medicine, University of Pennsylvania, Philadelphia, Pennsylvania, USA

${ }^{2}$ Ovarian Cancer Research Center, University of Pennsylvania, Philadelphia, Pennsylvania, USA
${ }^{3}$ School of Biosciences and Veterinary Medicine, University of Camerino, Camerino, Marche, Italy

\section{Twitter Augusto Amici @augusto.amici@unicam.it}

Acknowledgements We would like to thank Diana G Kattan, Francesco Marrese, and loannis Verginadis for their help with manuscript (DGK) and figures (FM) editing and immunofluorescence analysis (IV), respectively.

Contributors SP helped design the studies, performed the experiments, analyzed the data, and drafted the manuscript. AM and SR helped writing the manuscript and performed experiments. RP-L, MU-H, SB, AG, SP, and FC performed experiments. JGF edited the manuscript. AA assisted in study design. CK helped in conceiving the project and writing the manuscript. AF conceived the experiments, supervised the project, and wrote the manuscript.

Funding This work was supported by the NIH/NCl 5R01CA206012-04.

Competing interests None declared.

Patient consent for publication Not required.

Ethics approval All animal studies were approved by the IACUC and University Laboratory Animal Resources at the University of Pennsylvania. Mice were treated in accordance with University of Pennsylvania guidelines.

Provenance and peer review Not commissioned; externally peer reviewed.

Data availability statement No data are available. Not applicable.

Supplemental material This content has been supplied by the author(s). It has not been vetted by BMJ Publishing Group Limited (BMJ) and may not have been peer-reviewed. Any opinions or recommendations discussed are solely those of the author(s) and are not endorsed by BMJ. BMJ disclaims all liability and responsibility arising from any reliance placed on the content. Where the content includes any translated material, BMJ does not warrant the accuracy and reliability of the translations (including but not limited to local regulations, clinical guidelines, terminology, drug names and drug dosages), and is not responsible for any error and/or omissions arising from translation and adaptation or otherwise.

Open access This is an open access article distributed in accordance with the Creative Commons Attribution Non Commercial (CC BY-NC 4.0) license, which permits others to distribute, remix, adapt, build upon this work non-commercially, and license their derivative works on different terms, provided the original work is properly cited, appropriate credit is given, any changes made indicated, and the use is non-commercial. See http://creativecommons.org/licenses/by-nc/4.0/.

\section{ORCID iDs}

Stefano Pierini http://orcid.org/0000-0003-3082-1291

Francesca Costabile http://orcid.org/0000-0002-4784-4556

\section{REFERENCES}

1 Deng L, Liang H, Xu M, et al. Sting-Dependent cytosolic DNA sensing promotes radiation-induced type I interferondependent antitumor immunity in immunogenic tumors. Immunity 2014;41:843-52.

2 Nabet BY, Qiu Y, Shabason JE, et al. Exosome RNA Unshielding couples stromal activation to pattern recognition receptor signaling in cancer. Cell 2017;170:352-66.

3 Apetoh L, Ghiringhelli F, Tesniere A, et al. Toll-Like receptor 4dependent contribution of the immune system to anticancer chemotherapy and radiotherapy. Nat Med 2007;13:1050-9.

4 Demaria S, Coleman CN, Formenti SC. Radiotherapy: changing the game in immunotherapy. Trends Cancer 2016;2:286-94.

5 Reits EA, Hodge JW, Herberts CA, et al. Radiation modulates the peptide repertoire, enhances MHC class I expression, and induces successful antitumor immunotherapy. J Exp Med 2006;203:1259-71.

6 Garcia-Barros Met al. Tumor response to radiotherapy regulated by endothelial cell apoptosis. Science 2003;300:1155-9.

7 Chen F-H, Chiang C-S, Wang C-C, et al. Radiotherapy decreases vascular density and causes hypoxia with macrophage aggregation in TRAMP-C1 prostate tumors. Clin Cancer Res 2009;15:1721-9.

8 Park HJ, Griffin RJ, Hui S, et al. Radiation-Induced vascular damage in tumors: implications of vascular damage in ablative hypofractionated radiotherapy (SBRT and SRS). Radiat Res 2012;177:311-27.

9 El Kaffas A, Giles A, Czarnota GJ. Dose-Dependent response of tumor vasculature to radiation therapy in combination with sunitinib 
depicted by three-dimensional high-frequency power Doppler ultrasound. Angiogenesis 2013;16:443-54.

10 Patel SA, Minn AJ. Combination cancer therapy with immune checkpoint blockade: mechanisms and strategies. Immunity 2018;48:417-33.

11 Pierini S, Tanyi JL, Simpkins F. Ovarian granulosa cell tumor characterization identifies FOXL2 as an immunotherapeutic target. JCl Insight 2020:5.

12 Ugel S, Facciponte JG, De Sanctis F, et al. Targeting tumor vasculature: expanding the potential of DNA cancer vaccines. Cancer Immunol Immunother 2015;64:1339-48.

13 Facciponte JG, Ugel S, De Sanctis F, et al. Tumor endothelial marker 1-specific DNA vaccination targets tumor vasculature. J Clin Invest 2014;124:1497-511.

14 Rice J, Ottensmeier $\mathrm{CH}$, Stevenson FK. Dna vaccines: precision tools for activating effective immunity against cancer. Nat Rev Cancer 2008;8:108-20.

15 Rice J, Elliott T, Buchan S, et al. DNA fusion vaccine designed to induce cytotoxic $T$ cell responses against defined peptide motifs: implications for cancer vaccines. J Immunol 2001;167:1558-65.

16 Stevenson FK, Ottensmeier CH, Johnson P, et al. Dna vaccines to attack cancer. Proc Natl Acad Sci U S A 2004;101:14646-52.

17 Pierini S, Perales-Linares R, Uribe-Herranz M, et al. Trial Watch: DNA-based vaccines for oncological indications. Oncoimmunology 2017;6:e1398878

18 Pierini S, Fang C, Rafail S, et al. A tumor mitochondria vaccine protects against experimental renal cell carcinoma. J Immunol 2015;195:4020-7.

19 Cappuccini F, Bryant R, Pollock E, et al. Safety and immunogenicity of novel 5T4 viral vectored vaccination regimens in early stage prostate cancer: a phase I clinical trial. $J$ Immunother Cancer 2020;8:e000928.

20 Mennuni C, Calvaruso F, Facciabene A, et al. Efficient induction of T-cell responses to carcinoembryonic antigen by a heterologous prime-boost regimen using DNA and adenovirus vectors carrying a codon usage optimized cDNA. Int J Cancer 2005;117:444-55.

21 Ishizaki H, Song G-Y, Srivastava T, et al. Heterologous prime/boost immunization with p53-based vaccines combined with Toll-like receptor stimulation enhances tumor regression. $J$ Immunother 2010;33:609-17.

22 Facciabene A, Aurisicchio L, Elia L, et al. Dna and adenoviral vectors encoding carcinoembryonic antigen fused to immunoenhancing sequences augment antigen-specific immune response and confer tumor protection. Hum Gene Ther 2006;17:81-92.

23 McConkey SJ, Reece WHH, Moorthy VS, et al. Enhanced T-cell immunogenicity of plasmid DNA vaccines boosted by recombinant modified vaccinia virus Ankara in humans. Nat Med 2003;9:729-35

24 Brown SA, Surman SL, Sealy R, et al. Heterologous prime-boost HIV-1 vaccination regimens in pre-clinical and clinical trials. Viruses 2010;2:435-67.

25 Hallengärd D, Lum F-M, Kümmerer BM, et al. Prime-Boost immunization strategies against Chikungunya virus. J Virol 2014;88:13333-43.

26 Kim S-B, Ahn J-H, Kim J, et al. A phase 1 study of a heterologous prime-boost vaccination involving a truncated HER2 sequence in patients with HER2-expressing breast cancer. Mol Ther Methods Clin Dev 2015;2:15031.

27 Smith CL, Dunbar PR, Mirza F, et al. Recombinant modified vaccinia Ankara primes functionally activated CTL specific for a melanoma tumor antigen epitope in melanoma patients with a high risk of disease recurrence. Int J Cancer 2005;113:259-66.

28 Zhang $\mathrm{H}$, Liu C, Zhang F, et al. Muc1 and survivin combination tumor gene vaccine generates specific immune responses and anti-tumor effects in a murine melanoma model. Vaccine 2016;34:2648-55.

29 Liu C, Lu Z, Xie Y, et al. Soluble PD-1-based vaccine targeting MUC1 VNTR and survivin improves anti-tumor effect. Immunol Lett 2018;200:33-42.

30 Walker JM, Rolig AS, Charych DH, et al. NKTR-214 immunotherapy synergizes with radiotherapy to stimulate systemic $\mathrm{CD} 8^{+} \mathrm{T}$ cell responses capable of curing multi-focal cancer. J Immunother Cancer 2020;8:8.

31 Hillman GG, Reich LA, Rothstein SE, et al. Radiotherapy and MVAMUC1-IL-2 vaccine act synergistically for inducing specific immunity to MUC-1 tumor antigen. J Immunother Cancer 2017;5:4.

32 Uribe-Herranz M, Rafail S, Beghi S, et al. Gut microbiota modulate dendritic cell antigen presentation and radiotherapy-induced antitumor immune response. J Clin Invest 2020;130:466-79.

33 Zhao A, Nunez-Cruz S, Li C, et al. Rapid isolation of high-affinity human antibodies against the tumor vascular marker Endosialin/ TEM1, using a paired yeast-display/secretory scFv library platform. $J$ Immunol Methods 2011;363:221-32.
34 Gulley JL, Madan RA, Pachynski R, et al. Role of antigen spread and distinctive characteristics of immunotherapy in cancer treatment. $J$ Natl Cancer Inst 2017;109:109.

35 Huang AY, Gulden PH, Woods AS, et al. The immunodominant major histocompatibility complex class I-restricted antigen of a murine colon tumor derives from an endogenous retroviral gene product. Proc Natl Acad Sci U S A 1996;93:9730-5.

36 Takeda J, Sato Y, Kiyosawa H, et al. Anti-Tumor immunity against CT26 colon tumor in mice immunized with plasmid DNA encoding $\beta$-galactosidase fused to an envelope protein of endogenous retrovirus. Cell Immunol 2000;204:11-18.

37 Demaria S, Formenti SC. Role of T lymphocytes in tumor response to radiotherapy. Front Oncol 2012;2.

38 Ribas A, Butterfield LH, Glaspy JA, et al. Current developments in cancer vaccines and cellular immunotherapy. $J$ Clin Oncol 2003;21:2415-32.

39 Jeong $\mathrm{H}$, Hwang I, Kang SH, et al. Tumor-Associated macrophages as potential prognostic biomarkers of invasive breast cancer. $J$ Breast Cancer 2019;22:38-51.

40 Ugolini A, Tyurin VA, Tyurina YY, et al. Polymorphonuclear myeloidderived suppressor cells limit antigen cross-presentation by dendritic cells in cancer. JCl Insight 2020;5. doi:10.1172/jci.insight.138581. [Epub ahead of print: 06 Aug 2020].

41 Kim K-J, Kim J-H, Lee SJ, et al. Radiation improves antitumor effect of immune checkpoint inhibitor in murine hepatocellular carcinoma model. Oncotarget 2017;8:41242-55.

42 Maute RL, Gordon SR, Mayer AT, et al. Engineering high-affinity PD-1 variants for optimized immunotherapy and immuno-PET imaging. Proc Natl Acad Sci U S A 2015;112:E6506-14.

43 Uribe-Herranz M, Bittinger K, Rafail S, et al. Gut microbiota modulates adoptive cell therapy via CD8 $\alpha$ dendritic cells and IL-12. JCl Insight 2018;3:e94952.

44 Xiang B, Baybutt TR, Berman-Booty L, et al. Prime-Boost Immunization Eliminates Metastatic Colorectal Cancer by Producing High-Avidity Effector CD8 ${ }^{+}$T Cells. J Immunol 2017;198:3507-14.

45 H-J K, Kim Y-J, Kim Y-S. Immunogenicity and safety profiles of genetic vaccines against human HER-2/neu in cynomolgus monkeys. Gene Ther 2008;15:1351-60.

46 Hsieh C-L, Chen D-S, Hwang L-H. Tumor-Induced immunosuppression: a barrier to immunotherapy of large tumors by cytokine-secreting tumor vaccine. Hum Gene Ther 2000;11:681-92.

47 Bergers G, Javaherian K, KM L. Effects of angiogenesis inhibitors on multistage carcinogenesis in mice. Science 1999;284:808-12.

48 Wei Y-quan, Wang Q-ru, Zhao X. Immunotherapy of tumors with xenogeneic endothelial cells as a vaccine. Nat Med 2000;6:1160-6.

49 Okaji Y, Tsuno NH, Kitayama J, et al. Vaccination with autologous endothelium inhibits angiogenesis and metastasis of colon cancer through autoimmunity. Cancer Sci 2004;95:85-90.

50 Rafii S. Vaccination against tumor neovascularization: promise and reality. Cancer Cell 2002;2:429-31.

51 Hajari Taheri F, Hassani M, Sharifzadeh Z, et al. T cell engineered with a novel nanobody-based chimeric antigen receptor against VEGFR2 as a candidate for tumor immunotherapy. IUBMB Life 2019;71:1259-67.

52 Park J-S, Kim I-K, Han S, et al. Normalization of tumor vessels by Tie2 activation and Ang2 inhibition enhances drug delivery and produces a favorable tumor microenvironment. Cancer Cell 2016;30:953-67.

53 Huang Y, Yuan J, Righi E, et al. Vascular normalizing doses of antiangiogenic treatment reprogram the immunosuppressive tumor microenvironment and enhance immunotherapy. Proc Natl Acad Sci U S A 2012;109:17561-6.

54 Mpekris F, Voutouri C, Baish JW, et al. Combining microenvironment normalization strategies to improve cancer immunotherapy. Proc Natl Acad Sci U S A 2020;117:3728-37.

55 Wennerberg E, Vanpouille-Box C, Bornstein S, et al. Immune recognition of irradiated cancer cells. Immunol Rev 2017;280:220-30.

56 Golden EB, Demaria S, Schiff PB, et al. An abscopal response to radiation and ipilimumab in a patient with metastatic non-small cell lung cancer. Cancer Immunol Res 2013;1:365-72.

57 Postow MA, Callahan MK, Barker CA, et al. Immunologic correlates of the abscopal effect in a patient with melanoma. $N$ Engl $J$ Med 2012;366:925-31.

58 Vanpouille-Box C, Alard A, Aryankalayil MJ, et al. Dna exonuclease TREX1 regulates radiotherapy-induced tumour immunogenicity. Nat Commun 2017;8:15618.

59 Lee Y, Auh SL, Wang Y, et al. Therapeutic effects of ablative radiation on local tumor require CD8+ T cells: changing strategies for cancer treatment. Blood 2009;114:589-95. 
60 Twyman-Saint Victor C, Rech AJ, Maity A, et al. Radiation and dual checkpoint blockade activate non-redundant immune mechanisms in cancer. Nature 2015;520:373-7.

61 Galluzzi L, Buqué A, Kepp O, et al. Immunological effects of conventional chemotherapy and targeted anticancer agents. Cancer Cell 2015;28:690-714.

62 Maity A, Mick R, Huang AC, et al. A phase I trial of pembrolizumab with hypofractionated radiotherapy in patients with metastatic solid tumours. Br J Cancer 2018;119:1200-7.

63 Xu-Monette ZY, Zhang M, Li J, et al. Pd-1/Pd-L1 blockade: have we found the key to Unleash the antitumor immune response? Front Immunol 2017;8:1597.

64 Garcia-Diaz A, Shin DS, Moreno BH, et al. Interferon receptor signaling pathways regulating PD-L1 and PD-L2 expression. Cell Rep 2017:19:1189-201.

65 Colombo MP. On OX40 and PD-1 combination: why should OX40 be first in sequence? clin cancer Res. American Association for Cancer Research 2017;23:5999-6001.

66 Shrimali RK, Ahmad S, Verma V, et al. Concurrent PD-1 blockade negates the effects of OX40 agonist antibody in combination immunotherapy through inducing T-cell apoptosis. Cancer Immunol Res 2017:5:755-66.

67 Stern C, Kasnitz N, Kocijancic D, et al. Induction of CD4 $4^{(+)}$and $\left.C D 8^{(+}\right)$ anti-tumor effector $\mathrm{T}$ cell responses by bacteria mediated tumor therapy. Int J Cancer 2015;137:2019-28.

68 Maynard SK, Marshall JD, MacGill RS, et al. Vaccination with synthetic long peptide formulated with $\mathrm{CpG}$ in an oil-in-water emulsion induces robust E7-specific CD8 T cell responses and TC-1 tumor eradication. BMC Cancer 2019;19:540.

69 Chen L, Azuma T, Yu W, et al. B7-H1 maintains the polyclonal T cell response by protecting dendritic cells from cytotoxic T lymphocyte destruction. Proc Natl Acad Sci U S A 2018;115:3126-31.

70 Pierini S, Jordanov SH, Mitkova AV, et al. Promoter hypermethylation of CDKN2A, MGMT, MLH1, and DAPK genes in laryngeal squamous cell carcinoma and their associations with clinical profiles of the patients. Head Neck 2014;36:1103-8.

71 Facciabene A, De Sanctis F, Pierini S, et al. Local endothelial complement activation reverses endothelial quiescence, enabling T-cell homing, and tumor control during T-cell immunotherapy. Oncoimmunology 2017;6:e1326442. 